\title{
Linking the Response Properties of Cells in Auditory Cortex with Network Architecture: Cotuning versus Lateral Inhibition
}

\author{
Jaime de la Rocha, Cristina Marchetti, Max Schiff, and Alex D. Reyes \\ Center for Neural Science, New York University, New York, New York 10003
}

The frequency-intensity receptive fields (RF) of neurons in primary auditory cortex (AI) are heterogeneous. Some neurons have $\mathrm{V}$-shaped RFs, whereas others have enclosed ovoid RFs. Moreover, there is a wide range of temporal response profiles ranging from phasic to tonic firing. The mechanisms underlying this diversity of receptive field properties are yet unknown. Here we study the characteristics of thalamocortical (TC) and intracortical connectivity that give rise to the individual cell responses. Using a mouse auditory TC slice preparation, we found that the amplitude of synaptic responses in AI varies non-monotonically with the intensity of the stimulation in the medial geniculate nucleus (MGv). We constructed a network model of MGv and AI that was simulated using either rate model cells or in vitro neurons through an iterative procedure that used the recorded neural responses to reconstruct network activity. We compared the receptive fields and firing profiles obtained with networks configured to have either cotuned excitatory and inhibitory inputs or relatively broad, lateral inhibitory inputs. Each of these networks yielded distinct response properties consistent with those documented in vivo with natural stimuli. The cotuned network produced V-shaped RFs, phasic-tonic firing profiles, and predominantly monotonic rate-level functions. The lateral inhibitory network produced enclosed RFs with narrow frequency tuning, a variety of firing profiles, and robust non-monotonic rate-level functions. We conclude that both types of circuits must be present to account for the wide variety of responses observed in vivo.

Key words: primary auditory cortex; frequency tuning; intensity tuning; lateral inhibition; computational model; network dynamics; thalamocortical transformation

\section{Introduction}

Neurons in primary auditory cortex (AI) display a variety of complex firing behaviors in response to acoustic stimuli. As with most neurons in the auditory pathway, the responses of AI neurons are frequency tuned (Schreiner et al., 2000) and intensity modulated, with a substantial fraction exhibiting non-monotonic rateintensity functions (Evans and Whitfield, 1964; Brugge et al., 1969; Suga, 1977; Pfingst and O’Connor, 1981; Phillips et al., 1985, 1995; Schreiner et al., 1992). In addition, AI neurons exhibit a wide variety of receptive field (RF) shapes (e.g., V-shaped, ovoid, etc.) and temporal response profiles, ranging from phasic to tonic firing (Abeles and Goldstein, 1972; Volkov and Galazjuk, 1991; Recanzone, 2000; Chimoto et al., 2002; DeWeese et al., 2003; Wang et al., 2005; Hromadka et al., 2008). The neural cir-

\footnotetext{
Received Dec. 17, 2007; revised June 24, 2008; accepted July 16, 2008.

This work was supported by the Spanish Ministerio de Educación y Ciencia (J.d.I.I.) and National Institutes of Health. We thank Ann-Marie Oswald for careful reading of this manuscript and helpful comments and one anonymous referee for her/his very useful suggestions. We also thank Maria Ter-Mikaelian and Mal Semple for insightful discussions.

Correspondence should be addressed to Jaime de la Rocha, Center for Neural Science, New York University, 4 Washington Place, Room 809, New York, NY 10003. E-mail: jrocha@cns.nyu.edu.

C. Marchetti's present address: European Brain Research Institute, 00143 Rome, Italy. D0I:10.1523/JNEUROSCI.1789-08.2008

Copyright $\odot 2008$ Society for Neuroscience $\quad 0270-6474 / 08 / 289151-13 \$ 15.00 / 0$
}

cuitry underlying these response properties is essentially unknown.

Synaptic inhibition plays an important role in shaping the frequency-intensity RFs and the temporal patterns (Suga, 1995; Phillips et al., 2002; Oswald et al., 2006). Inhibition may be involved in (1) sharpening the frequency tuning (Katsuki et al., 1958; Suga and Manabe, 1982; Shamma and Symmes, 1985; Chen and Jen, 2000; Wang et al., 2002), (2) generating a nonmonotonic rate-intensity function (Greenwood and Maruyama, 1965; Suga, 1971; Young and Brownell, 1976; Phillips, 1990; Wu et al., 2006; Tan et al., 2007), and (3) enhancing response timing (Ojima and Murakami, 2002; Wehr and Zador, 2003).

The functional relationship between excitatory and inhibitory inputs is of some debate. Initial intracellular recordings in vivo indicated that the excitatory and inhibitory inputs are primarily cotuned such that both vary proportionally with frequency and intensity (Wehr and Zador, 2003; Zhang et al., 2003; Tan et al., 2004). However, recent studies demonstrated that inhibition is more broadly tuned than excitation with respect to frequency (Wu et al., 2008) and that there is an unbalanced recruitment of excitation and inhibition as intensity increases (Wu et al., 2006; Tan et al., 2007).

Here, using a combination of experiments and computational modeling, we study the features of the thalamocortical (TC) circuit that give rise to the response properties of AI neurons. We 
show, using auditory thalamocortical slices (Cruikshank et al., 2002; Rose and Metherate, 2005), that the postsynaptic potentials (PSPs) evoked in a large fraction of cortical cells vary nonmonotonically with the intensity of the thalamic stimulus. This suggests that AI circuitry provides a substrate (1) for transforming a monotonic TC input generated in the thalamus into a nonmonotonic cortical response, or alternatively (2) for enhancing non-monotonic inputs from the thalamus. We compared the response properties obtained in networks with cotuned inputs versus networks with classical lateral inhibition. The lateral inhibitory network (LIN) produced non-monotonic responses, generated ovoid RFs, sharpened frequency tuning, and exhibited a variety of dynamical behaviors ranging from tonic to purely phasic firing. In contrast, the cotuned network $(\mathrm{CON})$ produced essentially monotonic rate-level functions, V-shaped RFs, no sharpening of the frequency tuning curve, and predominantly phasic-tonic responses. To account for the variety of responses found in vivo, we suggest that both cotuned and lateral inhibition architectures exist in the auditory cortex.

\section{Materials and Methods \\ Network model}

The network model consisted of a layer of excitatory cells in thalamus (TH) that innervated a layer of excitatory (E) and inhibitory (I) neurons in cortex (see Fig. $2 a$, left). Without loss of generality, we used $N_{\mathrm{TH}}=400$ TH cells, $N_{\mathrm{E}}=800 \mathrm{E}$ cells, and $N_{\mathrm{I}}=200 \mathrm{I}$ cells (although see below, Experiments). In both layers, cells were arranged on a one-dimensional line representing the tonotopic axis. The position of each neuron determined its characteristic frequency $(\mathrm{CF})$; cells spanned 4 octaves (sufficiently large to avoid border effects) and were spaced at 0.005 octaves. Because of the translational symmetry of the network, the position of each cell was defined as the difference $\Delta f$ (in octaves) between the CF and the frequency of the tone presented. The input current $h_{\alpha}(\Delta f, t)$ to a cortical cell at $\Delta$ fin layer $\alpha$ at time $t$ was the sum of the TH, cortical E, and I contributions:

$$
\begin{gathered}
h_{\alpha}(\Delta f, t)=h_{\alpha \mathrm{TH}}(\Delta f, t)+h_{\alpha \mathrm{I}}(\Delta f, t)+h_{\alpha \mathrm{E}}(\Delta f, t) \\
h_{\alpha \beta}(\Delta f, t)=J_{\alpha \beta} \sum_{f} g_{\alpha \beta}(f-\Delta f) r_{\beta}(f, t),(\alpha=\mathrm{E}, \operatorname{Iand} \beta=\mathrm{TH}, \mathrm{E}, \mathrm{I}),
\end{gathered}
$$

where $J_{\alpha \beta}$ are the synaptic weights, $r_{\beta}(\Delta f, t)$ is the response of the neuron at $\Delta f$ in layer $\beta$ (see below), and $g_{\alpha \beta}(\Delta f)$ are the connectivity functions (the subindices $\alpha \beta$ are read as the input from presynaptic population $\beta$ to postsynaptic population $\alpha$ ). The sum on $f$ in Equation 2 was taken over all cells in the network. For simplicity, we assumed that connectivity functions depended only on the presynaptic population so that $g_{\alpha \beta}(\Delta f)=g_{\beta}(\Delta f)(\beta=\mathrm{TH}, \mathrm{E}, \mathrm{I})$. Thus, the connectivity was all-to-all with the synaptic strength determined by the product $J_{\alpha \beta} g_{\alpha}(\Delta f)$. All the connectivity functions were described by a Gaussian function:

$$
g_{\alpha}(\Delta f)=e^{-\frac{\Delta f^{2}}{2 \sigma_{\alpha}^{2}}}
$$

where the parameter $\sigma_{\alpha}$ set the spatial spread of $g_{\alpha}(\Delta f)$ along the tonotopic axis. The spatial convergence of thalamic projections, $\sigma_{\mathrm{TH}}$, was set to 0.05 octaves. The relative spread of the recurrent $\mathrm{E}$ and I cortical connections determined two types of networks: (1) LIN had $\sigma_{\mathrm{E}}=0.1, \sigma_{\mathrm{I}}$ $=0.3$ octaves with synaptic weights $J_{\mathrm{ETH}}=0.25, J_{\mathrm{ITH}}=0.2, J_{\mathrm{EE}}=0.55$, $J_{\mathrm{EI}}=-0.2, J_{\mathrm{IE}}=0.4 \mathrm{nA} / \mathrm{s}$; and (2) CON had $\sigma_{\mathrm{E}}=0.085, \sigma_{\mathrm{I}}=0.035$ octaves with $J_{\mathrm{ETH}}=1, J_{\mathrm{ITH}}=0.3, J_{\mathrm{EE}}=0.5, J_{\mathrm{EI}}=-1.25, J_{\mathrm{IE}}=0.4 \mathrm{nA} / \mathrm{s}$. Note that the main difference between the two networks were the parameters $J_{\mathrm{EI}}$ and $\sigma_{\mathrm{I}}$, specifying the efficacy and spread of inhibition.

The values of the parameters were chosen to approximate quantitatively values measured in vivo such as the following: overall mean firing rates of regular spiking cells $(\sim 10$ spikes/s) and inhibitory cells $(\sim 20-60$ spikes/s) (Hromadka et al., 2008); maximum sustained rates of $\sim 20-40$ spikes/s (Wang et al., 2005); phasic or phasic-tonic firing profiles with no activity ringing (i.e., multiple fading peaks) (Ulanovsky et al., 2003; Wang et al., 2005); thalamic cell firing at rates that are higher than those of excitatory cortical cells (Creutzfeldt et al., 1980); and RF widths of $\sim 1$ octave (Sally and Kelly, 1988; Kilgard and Merzenich, 1999). In the supplemental data (available at www.jneurosci.org as supplemental material), we confirmed that the results presented here were robust to variations of these parameter values.

\section{Stimulus}

Tone stimuli were parameterized by an intensity and frequency pair ( $I$, $f$ ). The tone was modeled with a stimulus function $s(\Delta f, t)$, which was a transient change in the input current to the thalamic layer of duration $T$ ( $T=150 \mathrm{~ms}$ in the model and $T=400-1000 \mathrm{~ms}$ in the experiments). The spatial profile of $s(\Delta f, t)$ was a Gaussian centered at the frequency $f$ with and amplitude set by the intensity $I$ (see Figs. $2 a, 7 f$ ):

$$
s(\Delta f, t)=A_{\text {in }}(I) \frac{-\Delta f^{2}}{2 \sigma_{i n}^{2}},
$$

where the amplitude varied with intensity as $A_{\text {in }}(I)=0.0125 I$ (with $A_{\text {in }}$ and $I$ in nanoamperes and decibels, respectively). The stimulus width was set to $\sigma_{\text {in }}=0.15$ octaves.

In the model, we also evaluated the suppressive effect of a masker tone of frequency and intensity ( $f_{\text {mask }}, I_{\text {mask }}$ ) on the response to another probe tone $\left(f_{\text {tone }}, I_{\text {tone }}\right)$. The two tones were presented simultaneously, and the thalamic input was given by the following sum:

$$
s(\Delta f)=\left[A_{\text {in }}\left(I_{\text {probe }}\right) e^{\frac{-\Delta f^{2}}{2 \sigma_{\text {in }}^{2}}}+A_{\text {in }}\left(I_{\text {mask }}\right) e^{\frac{-\left(\Delta f-f_{\text {makk }}\right)^{2}}{2 \sigma_{\text {in }}^{2}}}\right] .
$$

\section{Neuron model}

All cells in our computational model network were rate-based neurons (Wilson and Cowan, 1972; Ben-Yishai et al., 1995; Pinto et al., 2003). A single variable $r_{\beta}(\Delta f, t)$ represented the firing rate and determined the state of the each cell ( $\beta$ represents the layer index, $\Delta f$ the neuron position, and $t$ the time).

Thalamic cells. We did not model the temporal dynamics of the thalamic cells; rather, their firing rates were simply the transformation of the inputs: $r_{\mathrm{TH}}(\Delta f)=\phi_{\mathrm{TH}}(s(\Delta f))$. The transfer function $\phi_{\mathrm{TH}}(s)$ was based on in vitro recordings. We recorded from cells in the ventral division of the medial geniculate body (MGv), computed the average frequencycurrent $(f-I)$ curve, and fitted it with the second-order polynomial $f_{\mathrm{TH}}(I)=-118(I-0.11)^{2}+292(I-0.11)-3.4$ if $I>\theta_{\mathrm{TH}}$ and 0 otherwise ( $f_{\mathrm{TH}}$ is given in hertz and $I$ in nanoamperes; $\theta_{\mathrm{TH}}=0.12 \mathrm{nA}$ ). We increased the degree of concavity of $f_{\mathrm{TH}}(I)$ by defining the following:

$$
\phi_{\mathrm{TH}}(s)=\left\{\begin{array}{cc}
0 & s<\theta_{\mathrm{TH}} \\
z_{c}\left(f_{\mathrm{TH}}(s)\right) \cdot z_{b}(s) & s \geq \theta_{\mathrm{TH}}
\end{array}\right.
$$

where $z_{c}(x)$ is the logarithmic function $z_{c}(x)=100 \ln (c x+1)$ with $c$ the parameter setting the extra degree of concavity, and $z_{b}(s)=b\left(-s^{2}+s\right)+$ 1 is a non-monotonic auxiliary function with a degree of nonmonotonicity set by the parameter $b$ (see Fig. $7 i, j$ ). This parameterization allowed us to explore the different shapes observed in thalamic rate-level functions, which range from strictly monotonic, to saturating, and to non-monotonic (Rouiller et al., 1983) by systematically varying $c$ and $b$ (see Fig. $7 i, j$ ). The results presented here were reproduced qualitatively using other functional forms for $\phi_{\mathrm{TH}}(s)$ with a qualitatively similar nonlinearity.

For the in vitro network (see below), we modeled the response of the thalamic neurons as Poisson spike trains with rate $r_{\mathrm{TH}}(\Delta f)$ rather than modeling the thalamic transfer $\phi_{\mathrm{TH}}(s)$. The rate of the TH cells was set to the following:

$$
r_{\mathrm{TH}}(\Delta f)=A_{\mathrm{in}}(I) \frac{-\Delta f^{2}}{2 \sigma_{i n}^{2}(I)} .
$$

To mimic the effect of the nonlinear $\phi_{\mathrm{TH}}(s)$ used in the computational network, we introduce an ad hoc dependence of both the amplitude $A_{\text {in }}$ and the spread $\sigma_{\text {in }}$ on sound intensity $I$, which caused the thalamic activity $r_{\mathrm{TH}}(\Delta f)$ to saturate and to increase the spread along the tonotopic axes 
with growing $I$ (see Fig. $7 f-h$ ). In the experiments, we directly mimicked this effect by setting the following:

$$
\begin{gathered}
A_{\text {in }}(I)=\left\{\begin{array}{cl}
2 I & \text { if } I<30 d B \\
40 \mathrm{spks} / \mathrm{s} & \text { if } I>30 d B
\end{array}\right. \\
\sigma_{\text {in }}(I)=\left\{\begin{array}{cc}
1 / 8 \text { octvs. } & \text { if }<30 d B \\
-0.25+0.0125 \text { Ioctvs. } & \text { if }>30 d B
\end{array}\right.
\end{gathered}
$$

Thus, the amplitude increased linearly with the intensity (range $I=0-30$ $\mathrm{dB}$ ) and then saturated (range $I=30-80 \mathrm{~dB}$ ), leading to a linear increase of the lateral spread (range $I=30-80 \mathrm{~dB}$ ). The value of the transition point $I=30 \mathrm{~dB}$ was chosen freely to generate a strongly saturating function $r_{\mathrm{TH}}(\Delta f)$ consistent with many rate-level thalamic functions found in vivo (Rouiller et al., 1983). Larger values reduced the impact of saturation and diminished the degree of non-monotonicity of the cortical rate-level function, as occurred in the computational (see Fig. 7i,j). The results presented in this study were not sensitive to the exact functional dependence on $I$ of the inputs (Eqs. 4-6 for the computational network and Eqs. 7-9 for the in vitro network) and were robust to alternative parameterizations as long as they incorporated the spread of the stimulus with increasing intensity.

Cortical cells. The dynamics of the cortical rate-based model neurons (Wilson and Cowan, 1972) are given by the following:

$$
\tau_{\alpha} \frac{d r_{\alpha}(\Delta f, t)}{d t}=-r_{\alpha}(\Delta f, t)+\phi_{\alpha}\left(h_{\alpha}(\Delta f, t)\right),(\alpha=\mathrm{E}, \mathrm{I}) .
$$

The time constants, $\tau_{\mathrm{E}}=10 \mathrm{~ms}$ and $\tau_{\mathrm{I}}=7.5 \mathrm{~ms}$, reflected the faster kinetics of fast spiking (FS) cells relative to the regular spiking (RS) cells (Rose and Metherate, 2005; Cruikshank et al., 2007). $\phi_{\alpha}(s)(\alpha=\mathrm{E}$, I) was a threshold-linear transfer function with no saturation and was equal to $G\left(s-\theta_{\alpha}\right)$ if $s>\theta_{\alpha}(\alpha=\mathrm{E}, \mathrm{I})$ and 0 otherwise. The gain, $G=75$ spikes $\cdot \mathrm{s}^{-1} \cdot \mathrm{nA}^{-1}$, and thresholds, $\theta_{\mathrm{E}}=\theta_{\mathrm{I}}=0.05 \mathrm{nA}$, were obtained from approximate linear fits of the suprathreshold region of $f-I$ curves measured from pyramidal cells. Equation 10 was solved numerically using a standard Euler method.

Analysis of cell responses. In both the computational model network and the in vitro model network, cortical responses $r_{\alpha}(\alpha=\mathrm{E}, \mathrm{I})$ were defined by the average rate over the duration of the stimulus $T$. We quantified the phasic nature of the responses independently of overall magnitude using the index $P$ :

$$
P=\frac{r_{\text {peak }}-r_{\text {sust }}}{r_{\text {peak }}}
$$

where $r_{\text {peak }}$ and $r_{\text {sust }}$ were the maximum instantaneous rate and the sustained rate estimated as the average rate over a small window before the offset of the stimulus, respectively. Thus, $P$ ranges between 0 (tonic) and 1 (purely phasic), with values $\sim 0.5$ representing phasic-tonic.

\section{Experiments}

Surgical procedure and recordings. Thalamocortical auditory slices from postnatal days 14-20 mice were prepared as described by Cruikshank et al. (2002) and in accordance with guidelines of the New York University Animal Welfare Committee. Briefly, mice were anesthetized with halothane and decapitated. The brain was removed and placed in cold $(0-$ $4^{\circ} \mathrm{C}$ ) artificial CSF (ACSF) (in mm: $125 \mathrm{NaCl}, 25 \mathrm{NaHCO}_{3}, 25$ glucose, 2.5 $\mathrm{KCl}, 1.25 \mathrm{NaH}_{2} \mathrm{PO}_{4}, 2 \mathrm{CaCl}_{2}$, and $1 \mathrm{MgCl}_{2}$ ) bubbled with $95 \% \mathrm{O}_{2}, 5 \%$ $\mathrm{CO}_{2}$. Slices ( $500 \mu \mathrm{m}$ thickness) were cut at a $15^{\circ}$ angle from the horizontal plane with a Campden Instruments tissue slicer. Recordings were made from the "primary" slice (Cruikshank et al., 2002), chosen based on distance from the ventral surface.

During recordings, slices were perfused with $30-34^{\circ} \mathrm{C}$ ACSF (with $95 \% \mathrm{O}_{2}, 5 \% \mathrm{CO}_{2}$ ), and cells were identified with the aid of infrared video microscopy. Simultaneous whole-cell somatic recordings were made from a pyramidal neuron and an interneuron, both identified by their firing pattern and spike widths. Pipettes were filled with $130 \mathrm{~mm}$ K-gluconate, $5 \mathrm{~mm} \mathrm{KCl}, 10 \mathrm{~mm}$ phosphocreatine, $10 \mathrm{~mm}$ HEPES, $4 \mathrm{~mm}$ ATP-Mg, and $0.3 \mathrm{~mm}$ GTP at pH 7.3 (filled electrode resistances were in the range of 5-10 M $\Omega$ ), and recordings were performed under currentclamp conditions.

Synaptic potentials evoked with thalamic stimulation. Bipolar electrodes were used to deliver brief current pulses $(0.2 \mathrm{~ms})$ in MGv while performing simultaneous whole-cell recordings from at least two cells in layer 4 of AI. These cells were classified as excitatory RS cells or inhibitory FS cells, according to the firing patterns evoked with injected current steps. The stimulation intensity was first set to a low value that barely evoked a response and was then increased progressively (approximate range, 10$200 \mu \mathrm{A}$ ) to obtain a wide variety of amplitudes in the evoked PSP. Action potentials, evoked in some cells at high intensities, were removed from voltage traces using a median boxcar filter $(3 \mathrm{~ms})$, before averaging (10-15 trials). The response to a given intensity $I$ was quantified by the area of the PSP, $r(I)=\int_{0}^{T}\left(V(t)-V_{\text {rest }}\right) d t$, from the midpoint between the stimulus artifact and the PSP onset, to $T=50 \mathrm{~ms}$. The nonmonotonic index $m$ was defined as the ratio of the maximum response to the response at maximum intensity:

$$
m=\frac{r(\max [I])}{\max [r(I)]}
$$

Reconstructing network activity using an iterative procedure. The in vitro model network had the same qualitative structure as the computational network except that it contained only feedforward connections (ITH, $\mathrm{ETH}$, and EI). The connection between layers was sparse (see below) and random. The connectivity matrices was defined as $J_{\alpha \beta}\left(f_{i}, f_{j}\right)=1$ if cell at $f_{j}$ was connected to cell at $f_{i}$ and 0 otherwise, and the probability of connection was determined by the function $g_{\alpha \beta}\left(f_{i}-f_{j}\right)$ normalized to peak at 0.2 . In essence, the rate-based cells used in the computational network were replaced with neurons recorded in vitro. The network dynamics were then simulated using the iterative procedure described previously (Reyes, 2003). In step 1, the thalamic spike trains, described as $r_{\mathrm{TH}}(\Delta f, t)=\Sigma k \delta\left(t-t_{\Delta f}^{k}\right)$, with $t_{\Delta f}^{k}$ being the spike times of the cell at $\Delta f$ and $\delta(t)$ the Dirac $\delta$ function, were generated with a computer for each neuron according to a Poisson process with rate given by Equation 7. In step 2, to produce the input currents $h_{\mathrm{ETH}}(\Delta f, t)$ and $h_{\mathrm{ITH}}(\Delta f, t)$ for each recipient $\mathrm{E}$ and I neurons, respectively, the spike trains $r_{\mathrm{TH}}(\Delta f, t)$ were convolved and summed:

$$
h_{\alpha \mathrm{TH}}(\Delta f, t)=\sum_{f} J_{\alpha \mathrm{TH}}(\Delta f, f) \int d t^{\prime} A\left(t^{\prime}-t\right) r_{\mathrm{TH}}\left(f, t^{\prime}\right),(\alpha=\mathrm{E}, \mathrm{I}),
$$

where $A(t)$ gives the time course of the EPSC or IPSC [PSC: $A(t)$ $\left.=a\left(1-e^{\left.-t / \tau_{\text {rise }}\right)}\right) e^{-t / \tau_{\text {decay }}}\left(\tau_{\text {rise }}=1 \mathrm{~ms}, \tau_{\text {decay }}=2 \mathrm{~ms}\right)\right]$. The magnitude of the PSCs was set by the parameter $a$ to produce amplitudes of $\sim 0.8-1$ $\mathrm{mV}$ (Reyes et al., 1998). In step 3 , each of the currents $h_{\mathrm{ITH}}(\Delta f, t)$ was injected into an interneuron to obtain the output spike trains $r_{I}(\Delta f, t)$,which were saved. In step 4 , the spike trains $r_{I}(\Delta f, t)$ were convolved as in Equation 13 (with negative amplitude PSCs) and summed, to give the term $h_{\mathrm{EI}}(\Delta f, t)$, which represents the inhibitory inputs to the $\mathrm{E}$ cells. This was summed to the excitatory current to the E cells from the thalamus (Eq. 13 with $\alpha=\mathrm{E}$ ) to obtain $h_{\mathrm{E}}(\Delta f, t)=h_{\mathrm{ETH}}(\Delta f, t)+h_{\mathrm{EI}}(\Delta f, t)$. In step 5, finally, the total current $h_{\mathrm{E}}(\Delta f, t)$ was injected into a recorded pyramidal neuron to obtain the response $r_{\mathrm{E}}(\Delta f, t)$, and the procedure was repeated for each $\mathrm{E}$ cell in the cortical layer. The entire in vitro network simulation (from step 1 to step 5) was repeated for three to five trials (with different Poisson realizations of the thalamic firing), and the mean cortical rates were computed as the total number of spikes divided by the stimulus length, averaged over trials. Some experiments were also performed under dynamic clamp in which the input was delivered as conductance rather than current (Reyes, 2003).

\section{Results}

\section{Non-monotonic thalamocortical synaptic responses}

Using the TC brain slice containing auditory cortex and the thalamic nucleus MGv (Cruikshank et al., 2002), we recorded the 
a

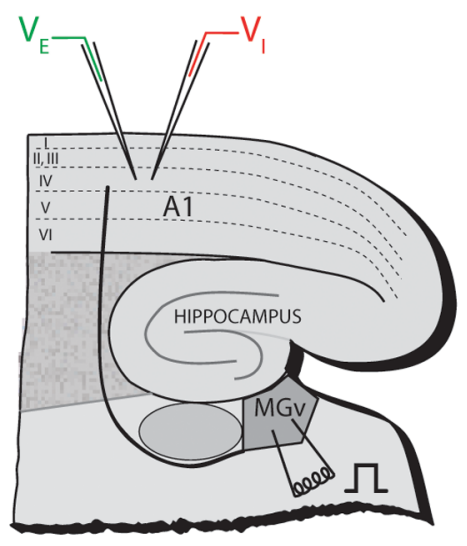

b

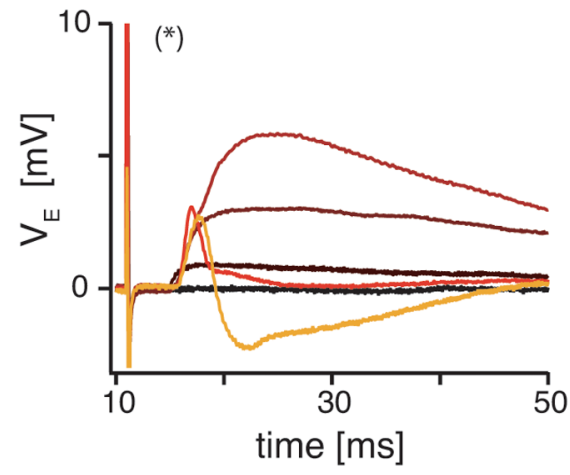

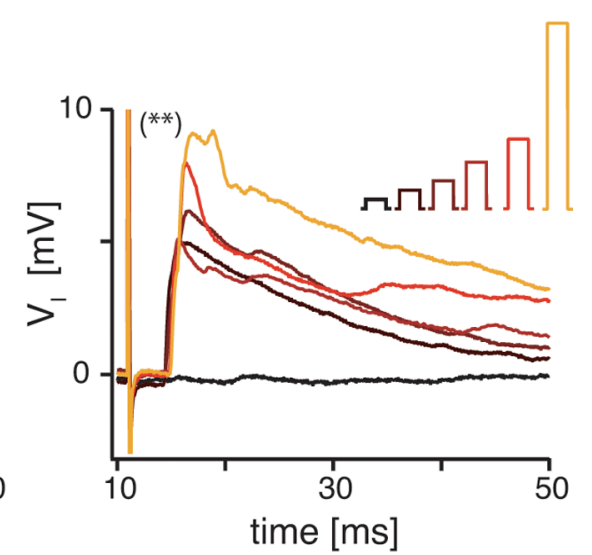

d
C

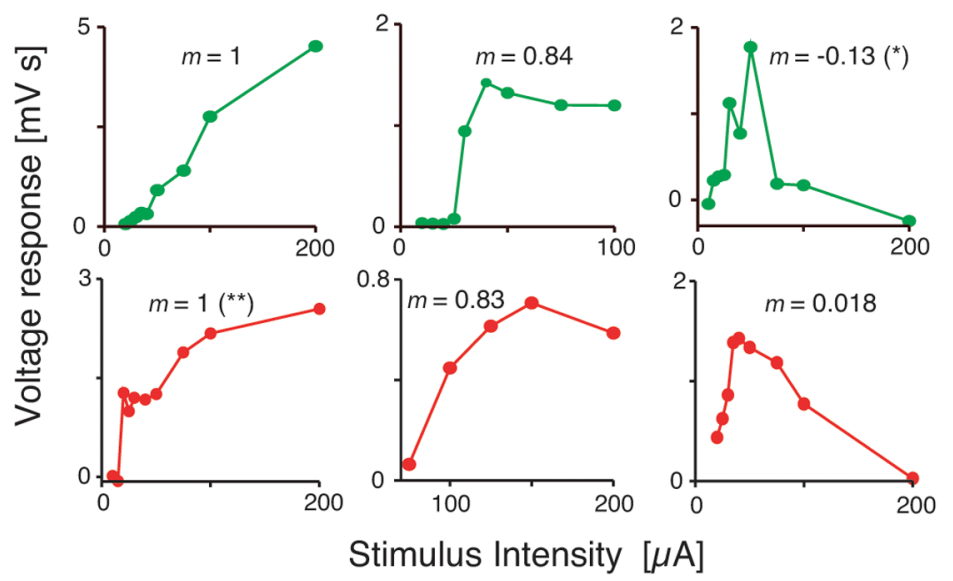

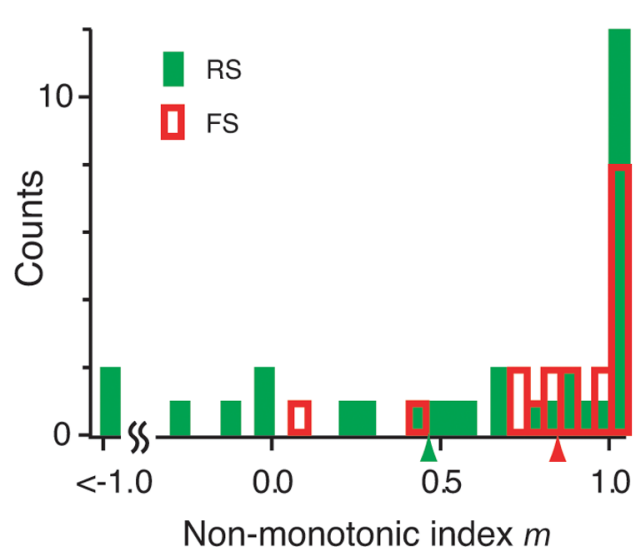

Figure 1. Postsynaptic potentials evoked with thalamic stimulation. $\boldsymbol{a}$, Schematic of thalamocortical auditory slice preparation. Brief stimulus pulses (200 $\mu$ s) were delivered to MGv and the PSPs recorded simultaneously in RS and FS cells in layer 4. $\boldsymbol{b}$, PSPs (averaged from 10 - 15 trials) evoked at different stimulus intensities (10, 20,30, 50, 75, and 200 $\mu$ A; see inset color code) from an RS (left) and an FS (right) cell, simultaneously recorded. Action potentials were digitally filtered from voltage traces (see Materials and Methods). c, PSP area (calculated over a window of $50 \mathrm{~ms}$ from PSP onset) versus stimulus intensity for three RS cells (top row) and three FS cells (bottom row). Some cells (middle and right columns) showed distinct non-monotonic curves. Cells indicated with ${ }^{*}$ and ${ }^{* *}$ were those shown in $\boldsymbol{a} . \boldsymbol{d}$, Population histogram of the non-monotonicity index $m$ (Eq. 12), defined as the ratio of the response at maximum intensity to the maximum response. A larger fraction of RS cells (green) displayed non-monotonic behavior (15 of 33 RS vs 4 of 19 FS cells showed $m<0.75$ ) and overall had a lower mean $m$ ( 0.46 for RS vs 0.84 for FS; triangles).

PSPs evoked in layer IV neurons by a brief extracellular stimulus delivered to MGv (Fig. 1a) (see Materials and Methods). The stimulation intensity was first set to a minimal value that barely evoked a response and then increased progressively. Fig. $1 b$ shows the average PSPs evoked in a simultaneously recorded excitatory RS cell (left panel) and inhibitory FS cell (right panel) with increasing stimulus intensity. Within this range, the response of the two cells differed substantially; in particular, the PSP evoked in the RS at high-intensity stimulation exhibited brief depolarization followed by a prolonged hyperpolarization, whereas those evoked in the FS cell remained primarily depolarizing. To quantify the changes in PSP shapes with intensity, we plotted the area under the voltage trace from rest value over a $50 \mathrm{~ms}$ window versus the stimulus intensity for both RS (Fig. $1 c$, top row) and FS cells (Fig. $1 c$, bottom row). The non-monotonic index, $m$ (Eq. $12)$, for each cell type ranged from strictly monotonic $(m=1)$ (Fig. $1 d$, left) to mildly and strongly non-monotonic (Fig. $1 d$, right). Overall, almost half (15 of 33) of the RS cells recorded showed $m<0.75$, compared with only 4 of 19 FS cells (Fig. 1d). Furthermore, high-intensity stimulation produced predominantly inhibitory synaptic responses in RS (8 of 32) and FS (2 of 19) cells with (Fig. $1 b$, left) or without (data not shown) an excitatory component.
The non-monotonicity of the TC PSPs observed in our experiments is unlikely to be inherited from the thalamus. The short latency of the EPSPs suggests direct activation of thalamic fibers and therefore excludes the involvement of inhibition to TCs from the reticular formation. Even if the action potential were triggered at the TC soma, some time would be required to recruit inhibitory cells; any inhibition would arrive too late to affect the action potential that is already propagating toward the cortex. Moreover, TC stimuli often evoked PSPs that increased monotonically in the FS cell and non-monotonically in a simultaneously recorded RS cell (see example in Fig. $1 b ; c,{ }^{*} ; c,{ }^{* *}$ ). Thus, it is unlikely that the TC inputs in our experiments were themselves non-monotonic.

These results suggest that local cortical inhibition plays an important role in shaping the synaptic inputs from the thalamus. At the very least, inhibition could enhance the non-monotonicity of rate-level response functions of cortical cells that may have been inherited from thalamus.

\section{Thalamocortical network models}

To study the mechanisms underlying the basic response properties of AI cells, we constructed a network model consisting of a layer of excitatory $\mathrm{TH}$ cells that projected onto a cortical layer 


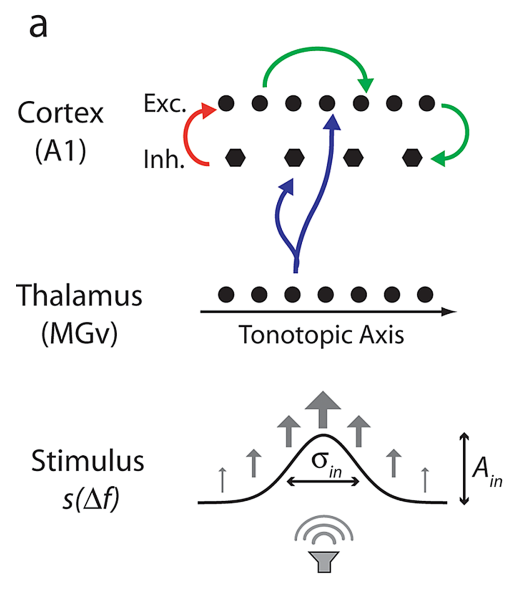

b
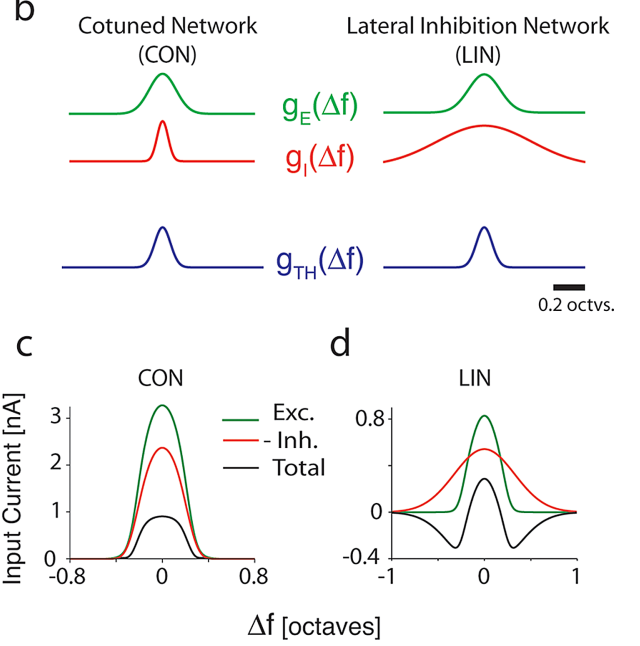

Figure 2. Implementation of cotuned and lateral inhibitory networks. $\boldsymbol{a}$, Schematic of the thalamocortical network model. Cells in the TH and cortical layers are spatially arranged along a tonotopic axis. The cortical layer is composed of E and I cells. TC afferents formed connections with $E$ and I cells (blue arrows), I cells were connected to $E$ cells (red), and $E$ cells are connected to $E$ and I cells (green). Inputs to thalamus during the presentation of a tone were modeled as a Gaussian distributed current $s(\Delta f)$ with amplitude $A_{\text {in }}$ and width $\sigma_{\text {in }}, \boldsymbol{b}$, CON and LIN are defined by connectivity functions, $g_{\alpha}(\Delta f)(\alpha=\mathrm{TH}$, E, l; color code matched with $\boldsymbol{a}$ ). In the LIN, the connectivity function for inhibition is wider than that for TC or the E, whereas in the CON it is slightly narrower. $\boldsymbol{c}, \boldsymbol{d}$, Synaptic currents generated in a reference $E$ cell in the cortical layer. In the $\mathrm{CON}$, the excitation and inhibition vary proportionally with increasing $\Delta f$, and the total current is approximately Gaussian (black in c). In the LIN, the tuning of inhibitory inputs is broader so that the total current has a Mexican hat shape (black in $\boldsymbol{d}$ ).

containing both $\mathrm{E}$ and I cells (Fig. 2a). In both layers, cells were arranged one dimensionally on a line representing the tonotopic axis (Fig. 2a). The position of each neuron on the axis was determined by the distance $\Delta f$, in octaves, between its CF and the frequency of the tone presented (see Materials and Methods). Thalamic neurons innervated $\mathrm{E}$ and I cells (Fig. $2 a$, blue arrows), both of which were connected recurrently (Fig. $2 a$, green and red arrows). The connection between each type of neuron was determined by $g_{\alpha}(\Delta f)$, which represents the synaptic strength between a postsynaptic cortical cell and a presynaptic cell of type $\alpha$ (with $\alpha=\mathrm{TH}, \mathrm{E}$, or I) located at a distance $\Delta$ falong the tonotopic axis (Fig. 2b). These functions are Gaussians such that neurons receive the majority of their inputs from neurons with similar CFs (Fig. 2b).

We examined two different realizations of the network: (1) the computational model network, using rate-based neurons with very simple dynamics (Wilson and Cowan, 1972; Ben-Yishai et al., 1995; Pinto et al., 2003) and (2) the in vitro model network, using an iterative process that uses the responses from recorded neurons to replicate the activity of a large population of neurons (Reyes, 2003) (see Materials and Methods).

To simulate the responses of thalamic neurons to a tone stimulus, a step of current, $s(\Delta f)$, was delivered to thalamic layer. The spatial profile along the tonotopic axis was Gaussian (Fig. $2 a$, bottom). The position of the peak on the axis corresponded to the tone frequency so that the magnitude of current into a particular cell along the tonotopic axis decreased with increasing $\Delta f$ (Eq. 4$)$. The $s(\Delta f)$ gave rise to the thalamic firing rate response, $r_{\mathrm{TH}}(\Delta f)$, via a thalamic transfer function $\phi_{\mathrm{TH}}(s)$, that is, $r_{\mathrm{TH}}(\Delta f)=$ $\phi_{\mathrm{TH}}(s(\Delta f))$ (see Materials and Methods).

We studied two networks that were defined based on the frequency tuning of the total excitatory $(\mathrm{TH}+\mathrm{E})$ versus inhibitory (I) inputs onto an E cell. In the CON, both excitatory and inhibitory inputs varied proportionally with $\Delta f$ (Fig. $2 c$ ). In the LIN, the inhibitory input had a broader frequency tuning than the excitatory input (Fig. 2d). These requirements for frequency tuning held at all intensities. Note that the definition of the CON and the LIN is functional (the tuning of the inputs) and not anatomical (the connectivity functions). There are several possible architectures (i.e., connectivity schemes) that can give rise to each network. However, the differences in receptive field properties and temporal firing profiles between the CON and LIN, summarized in Table 1, did not depend substantially on the details of the architecture. For this reason, the analysis will be done using one specific architecture for each functional network, and we will address separately those aspects that depend on the architecture (see supplemental data, available at www.jneurosci.org as supplemental material).

\section{Cotuned network}

The most important connectivity parameter that determined whether the $\mathrm{CON}$ or LIN occurred was the spread of the inhibitory connections $g_{I}(\Delta f)$ : essentially, for a given stimulus width $\sigma_{\text {in }}$ (Fig. $2 a$ ) and a fixed thalamocortical connectivity, narrower $g_{I}(\Delta f)$ yielded a CON, whereas broader $g_{I}(\Delta f)$ produced an LIN (supplemental Fig. S3, available at www.jneurosci.org as supplemental material). Interestingly, in a simple network like the one considered (Fig. 2a), cotuning or lateral inhibition were the dominant regimens under physiological conditions, implying that no fine-tuning of the parameters was needed to obtain either of the two network types (supplemental Fig. S3c, available at www.jneurosci.org as supplemental material).

Receptive fields obtained with the cotuned network

RFs of thalamic and cortical cells were constructed by systematically varying tone intensity and frequency and computing the resultant response rates. We set the thalamic cells to have a standard V-shaped RF (Fig. 3a) and a monotonic concave rate-level function (Fig. 3e, blue curve). The effects of non-monotonic thalamic rate-level functions are considered below (see Fig. $7 i, j$ ). The V-shape of the thalamic RF was obtained as a consequence of the "iceberg" effect produced by the firing threshold of the thalamic cells acting on a Gaussian stimulus input.

In the $\mathrm{CON}$, both $\mathrm{E}$ and I cells inherited the same $\mathrm{V}$-shape in their RFs, had the same $f$-tuning width as the TH cells (Fig. $3 f$ ), and exhibited the same monotonic thalamic rate-level function (Fig. 3e). This occurred because excitation and inhibition were recruited in a balanced (i.e., proportional) manner with increasing intensity (Fig. 3d). This allowed the frequency cotuning of $\mathrm{E}$ and I inputs to hold over a wide range of intensities (data not shown).

We also explored whether the nonlinearity imposed by the inhibitory threshold leads to an unbalanced recruitment of inhibition in the CON (Phillips, 1988; Phillips et al., 1995). For this, we considered the case in which the inhibitory threshold was disproportionably larger than the excitatory threshold, $\theta_{\mathrm{I}} \gg \theta_{\mathrm{E}}$, as opposed to above in which $\theta_{\mathrm{I}}=\theta_{\mathrm{E}}$. This configuration produced rate-level curves with moderate degrees of nonmonotonicity and a large asymmetry between the rising and de- 
Table 1. Summary of the response properties of cells in the CON and in the LIN

\begin{tabular}{|c|c|c|}
\hline & CON cells & LIN cells \\
\hline$f$ tuning of the total input current & Gaussian-like (Fig. 2c) & Mexican-hat-shaped (Fig. 2d) \\
\hline $\begin{array}{l}\text { Intensity dependence of input currents (when } \\
\text { TH current is monotonic) }\end{array}$ & E and I currents increase monotonically and proportionally (Fig. 3d) & $\begin{array}{l}\text { E current varies non-monotonically, } \\
\text { whereas I current increases monotonically (Figs. 5d,7e) }\end{array}$ \\
\hline Shape of the response RF & V-shaped (E, I) (Fig. 3b,c) & Ovoid (E) and narrow V-shaped (I) (Fig. 5b,c) \\
\hline Dynamics of response RF & Constant V shape (Fig. 4g) & $\begin{array}{l}\text { Dynamic transition from V at the onset, to narrow ovoid at the sus- } \\
\text { tained (Fig. } 6 g \text { ) }\end{array}$ \\
\hline Suppressive RF & Overlapping V-shaped (data not shown) & Surrounding W-shaped (Fig. 5g) \\
\hline Response $f$ tuning of $E$ cells & Same as $r_{\mathrm{TH}}(\Delta f)($ Fig. $3 f)$ and same as $h_{\mathrm{TH}}(\Delta f)$ (data not shown) & $\begin{array}{l}\left.\text { Sharpening with respect to } r_{\mathrm{TH}}(\Delta f) \text { (Fig. } 5 f\right) \text { and with respect to } \\
h_{\mathrm{TH}}(\Delta f) \text { (data not shown) }\end{array}$ \\
\hline Rate-level function & Shape inherited from TH, i.e. $m_{\mathrm{E}}=m_{\mathrm{TH}}$ (Fig. 3e) & $\begin{array}{l}\text { E cells are strongly non-monotonic and I cells slightly non-monotonic } \\
\text { (Fig. 5e). Sharpening of the TH rate-level function, i.e., } m_{\mathrm{E}}<m_{\mathrm{TH}} \\
\text { (Fig. } 7 i_{1} \text { ) }\end{array}$ \\
\hline Response temporal pattern & Invariant with stimulus, e.g., $P_{\mathrm{E}} \sim 0.5$ (Fig. $4 A a$ ) & $\begin{array}{l}\text { Depends on stimulus: E pattern varies with increasing intensity from } \\
\text { tonic to phasic, whereas I pattern varies from tonic to phasic-tonic } \\
\text { (Fig. } 6 \mathrm{Aa} \text { ) }\end{array}$ \\
\hline $\begin{array}{l}\text { Response of E cells to uniform input (broad } \\
\text { band noise) }\end{array}$ & Similar to a pure tone (data not shown) & $\begin{array}{l}\text { No response (supplemental Fig. S5d, available at www.jneurosci.org } \\
\text { as supplemental material) }\end{array}$ \\
\hline
\end{tabular}

caying edges (supplemental data, Fig. S4d, available at www.jneurosci.org as supplemental material). The ability of the CON to produce non-monotonic rate-level functions vanished as the excitatory and inhibitory thresholds became similar (supplemental Fig. S4d, available at www. jneurosci.org as supplemental material).

Response dynamics in the CON

We classified the temporal profiles of the responses with the index $P$ (Eq. 11), which ranged from 0 for tonic firing to 1 for purely phasic firing. The map of $P$ shows that responses had the same phasic-tonic profile $(P \sim 0.5)$ in response to most stimuli and were phasic only for tones with large $\Delta f$, in which almost no response was produced (Fig. 4Aa). When stimulated with a tone at $\mathrm{CF}$ and moderate intensity (Fig. $4 A a, \star$ ), both $\mathrm{E}$ and I responses were phasic-tonic, although the I cell reached a higher steady-state rate and had a substantially smaller $P$ value (Fig. $4 A c, P$ values by the curves). The same phasic-tonic response pattern was maintained at all intensities though the rates differed (Fig. $4 A b-A d$ ). Similarly, as $\Delta f$ increased (Fig. $4 A a, \mathbf{O}$ ), the phasic and tonic components of the $\mathrm{E}$ and I cell rates decreased proportionately (Fig. 4Ae). The phasic-tonic behavior occurred because (1) the inhibitory feedforward input into the E cell was delayed relative to the excitatory input, which derives directly from the thalamic drive, whereas inhibition must go through an extra synapse (data not shown), and (2) because feedback inhibition is recruited by cortical excitation so that the change in rate of the I cells tends to lag behind the change in the rate of E cells. Increasing the inhibitory synaptic strength $J_{\mathrm{EI}}$ causes a general decrease in the response rates and a substantial increase in $P$ (data not shown), in line with what has been observed in vivo in the

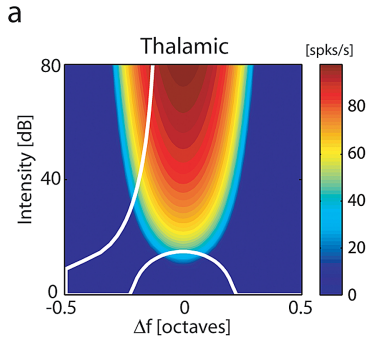

b
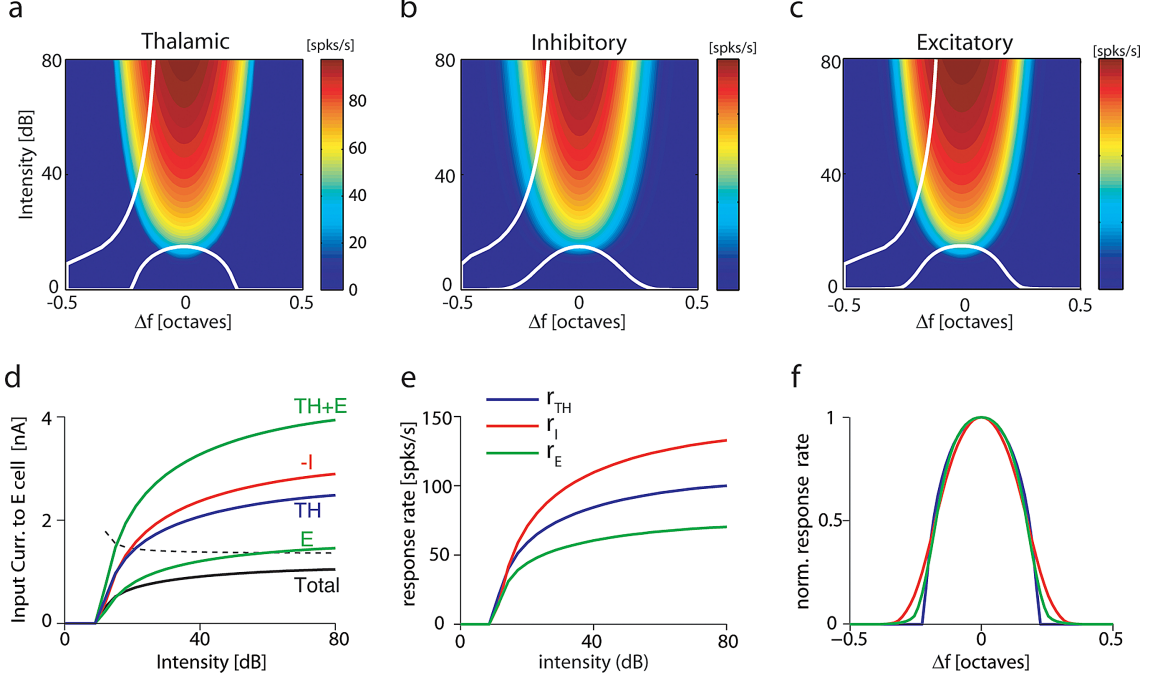

igure 3. Receptive field properties in the CON. $\boldsymbol{a}-\boldsymbol{c}$, RFs of $\mathrm{TH}, \mathrm{I}$, and $\mathrm{E}$ cells calculated by varying stimulus frequency and respectively, of each panel. $\boldsymbol{d}$, Input currents into a reference E cell show a proportional recruitment of excitation (green curves) and inhibition (red curve) with increasing intensity. The ratio between the excitatory input and the inhibitory input (dashed line) is approximately constant. $\boldsymbol{e}, \boldsymbol{f}$, Rate-level functions $(\boldsymbol{e})$ and frequency tuning curves $(\boldsymbol{f})$ show that cortical $\mathrm{E}$ and I cells mimic the behavior of TH cells. Intensity was $40 \mathrm{db}(\boldsymbol{f})$ and $f=C \mathrm{~F}(\boldsymbol{e})$. Legend in $\boldsymbol{e}$ also applies to $\boldsymbol{f}$.

presence of anesthesia. In general, the strength of inhibition determined the response pattern, which in the $\mathrm{CON}$ was approximately maintained at all intensities and $\Delta f$.

The extent of response across the entire E population maintained the same spread along the tonotopic axis for the duration of the stimulus; the breadth of the transient component (25-50 $\mathrm{ms})$ was only slightly greater than that of the steady-state $(>100$ ms) component (Fig. 4Af). As a result, the shape of the RF was similar whether computed over the transient component (from 0 to $50 \mathrm{~ms}$ ) or during the late stationary component of the response (from 100 to $150 \mathrm{~ms}$ ) (Fig. $4 g$ ).

To test the generality of the results obtained with the computa- 


\section{A. Computational Network}

a
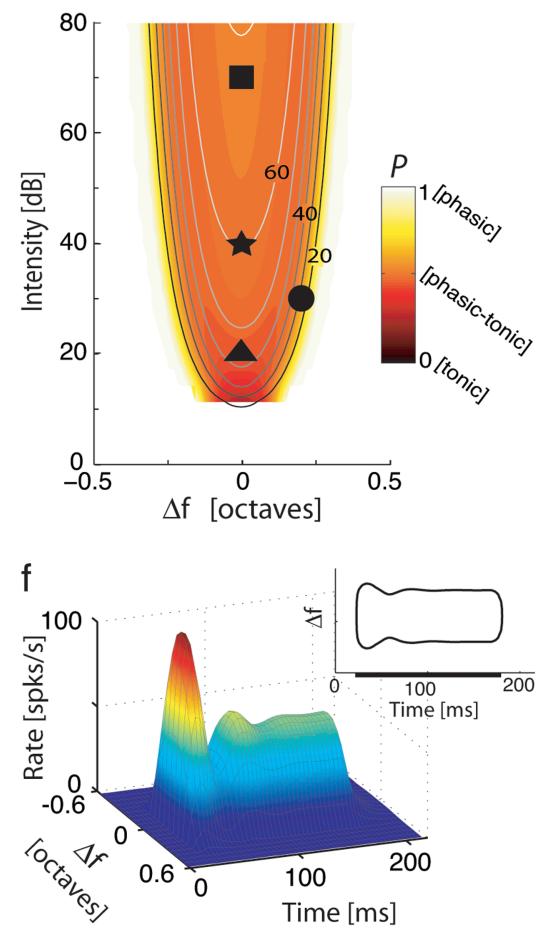

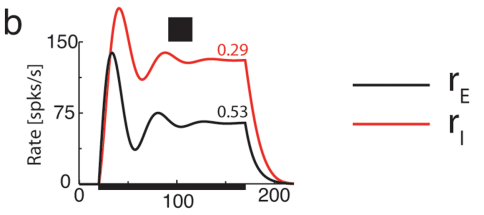

C

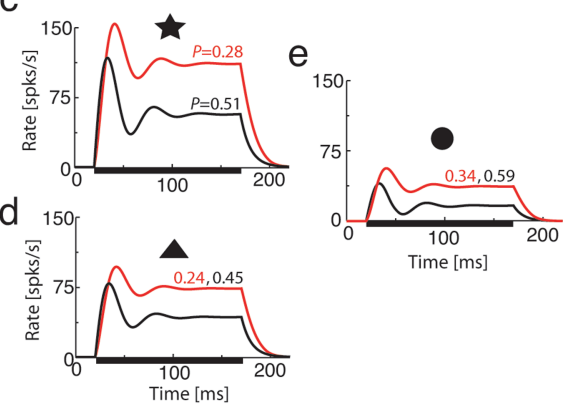

9

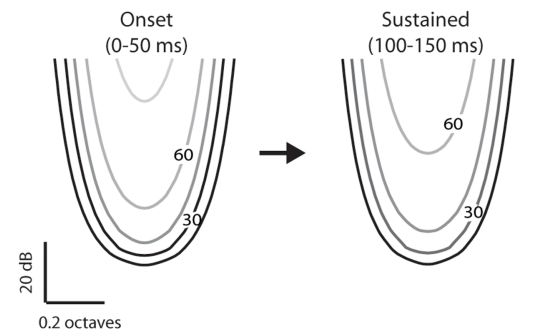

\section{B. In-vitro Network}

a

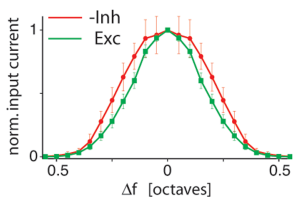

Figure 4. Response dynamics in the CON. $A$, Computational network. $\boldsymbol{a}$, Map of $P$ index (color) superimposed on RF of an $E$ cell (contour lines). The $P$ index (Eq. 11) quantifies the relative magnitude of the transient and sustained components of the response profile. Except at large $\Delta f$, the response is always phasic-tonic. Symbols represent responses highlighted in $\boldsymbol{b}$ - $\boldsymbol{e}$. $\boldsymbol{b}$ - $\boldsymbol{d}$, Responses produced by tones at $(F$ and high, moderate, and low intensity $(\boldsymbol{\square}, \star$, and $\mathbf{\Delta}$ in $\boldsymbol{a}$, respectively) are approximately scaled versions of the same phasic-tonic pattern in $E$ cells $\left[r_{E}(t)\right.$, black $]$. The responses of the I cells $\left[r_{1}(t)\right.$, red] were more tonic with a weak phasic component (see $P$ values by the curves). The thick horizontal line in all plots shows the tone presentation interval.e, Increasing $\Delta f$ $\left(-\right.$ in $\boldsymbol{a}$ ) decreases both $r_{\mathrm{E}}(t)$ and $r_{1}(t)$ with little variation of the pattern. $\boldsymbol{f}$, Response pattern of the entire E population to a $40 \mathrm{~dB}$ tone. Inset shows the iso-rate contour line at half-maximum. $\boldsymbol{g}$, Iso-rate contour lines of the RF of an $\mathrm{E}$ cell computed during the onset (interval, $0-50 \mathrm{~ms}$ ) and sustained (interval, $100-150 \mathrm{~ms}$ ) parts of the response. $\boldsymbol{B}$, In vitro model network. $\boldsymbol{a}$, Normalized average excitatory and inhibitory currents into an Ecell show cotuning. $\boldsymbol{b}$, PSTHs (left) and representative voltage traces (right) of an $E$ cell as frequency deviates from CF. As in the computational model, increasing $\Delta f$ scales down the entire response. Parameters in Aa-Ae are as follows: $T=150 \mathrm{~ms} ;(\Delta f, I)=(0,40)(\star),(0,70)(\mathbf{\square}),(0,20)(\mathbf{\Delta}),(0.4,20)(\mathbf{O})$, all in octaves and decibels. Labels on contour lines in $\boldsymbol{a}$ indicate firing rate in spikes per second. Parameters in $\boldsymbol{B} \boldsymbol{a}, \boldsymbol{B} \boldsymbol{b}$ are as follows: $T=360 \mathrm{~ms} ; \Delta f=0,0.1,0.2$, and 0.3 octaves; PSTH bin size, $5 \mathrm{~ms}$ ( $\boldsymbol{b}$, left); $\Delta f=0,0.2$, and 0.35 octaves ( $\boldsymbol{b}$, right).

tional rate-based model neurons, we used the iteratively constructed network (ICN) in which responses of cells recorded in vitro were used to reconstruct the activity of the entire CON network (Reyes, 2003). Because the iterative simulations were performed with recorded RS and FS cells, the ICN inherently contains the intrinsic membrane nonlinearities of a cortical cell such as the spike generation mechanism, refractoriness, and response adaptation (Fig. $4 B c$ ). Despite the large differences between the in vitro and computational implementation of the $\mathrm{CON}$, we reproduced the stimulusindependent phasic-tonic nature of the responses: when the currents injected to each cell were cotuned in $f$ (Fig. $4 \mathrm{Ba}$ ), responses were always phasic-tonic. The overall response decreased with increasing $\Delta f$, as shown by both peristimulus time histograms (PSTHs) and voltage traces (Fig. $4 B b$ ). This suggests that this is a property of the CON architecture and does not depend substantially on the particularities of the neuronal properties.

\section{Lateral inhibitory network \\ Architecture yielding a lateral inhibitory network}

The LIN can be constructed using three simple and not mutually exclusive connectivity schemes. In one scheme, cortical I cells send their axons laterally to neighboring columns with different CFs (Fig. $2 b$, right). In another scheme, I cells receive substantial convergent inputs from thalamic cells with more distant CFs (supplemental Fig. S6a, available at www. jneurosci.org as supplemental material). In these two architectures, lateral inhibition is recruited by TC inputs (i.e., feedforward inhibition) and therefore both produce qualitatively the same results. We focus on the first scheme, which is in line with the in vitro data (Fig. 1) and with indirect evidence from in vivo experiments, which suggest that most of the inhibition received in response to a short tone is feedforward (Wehr and Zador, 2003; Tan et al., 2004).

A third scheme produces lateral inhibition via feedback from cortical E cells, which projects laterally to I cells in neighboring columns with different CFs (supplemental Fig. S8a, available at www. jneurosci.org as supplemental material). This scheme, however, is not as effective as the feedforward-based LIN in reproducing cortical responses (supplemental data, available at www.jneurosci.org as supplemental material).

\section{Receptive fields obtained with the LIN}

Using the same thalamic V-shaped RFs used for the CON, the LIN network generated ovoid RFs in the E cells (Phillips et al., 1985, 1995; Calford and Semple, 1995; Heil and Irvine, 1998; Jen, 2002; Sutter and Loftus, 2003; Sadagopan and Wang, 2008) because of the unbalanced recruitment of excitation and inhibition with increasing intensity (Fig. $5 d$; see below). The intracortical dynamics of the LIN generated non-monotonic rate-level functions (Fig. 5e) and greatly sharpened the frequency tuning of E cells (Fig. $5 f$ ), indicating that lateral inhibition is a suitable mechanism for the response suppression at high intensities and at frequency regions flanking the CF (Sutter et al., 1999; Phillips et al., 2002; Wang et al., 2002; Wu et al., 2008). Moreover, unlike the CON, the RF of E cells was much narrower than the thalamic synaptic input RF (data not shown) in agreement with in vivo data (Liu et al., 2007). 
The inhibitory RF remained V-shaped (Fig. $5 b$ ) but did exhibit some frequency sharpening (Fig. $5 f$ ) and very modest nonmonotonicity at high intensities (Fig. 5e). These results are consistent with the evoked PSPs being more non-monotonic in RS cells than in FS cells (Fig. 1d). Inhibitory cells also showed broader RFs than excitatory cells (Fig. $5 b, c$ ), in contrast to the CON (Fig. 3b,c) and in agreement with recent in vivo experiments (Atencio and Schreiner, 2008; Wu et al., 2008).

The LIN reproduced two-tone suppressive RFs similar to what has been documented in vivo (Shamma and Symmes, 1985; Calford and Semple, 1995; Brosch and Schreiner, 1997; Sutter et al., 1999; Sutter and Loftus, 2003). In those experiments, the response to a probe tone at the $\mathrm{CF}$ and best intensity was suppressed by a simultaneous tone played at frequency $f_{\text {mask }}$ and intensity $I_{\text {mask }}$ (Ehret and Merzenich, 1988; Calford and Semple, 1995; Brosch and Schreiner, 1997; Sutter et al., 1999; Sutter and Loftus, 2003). The LIN produced a suppressive field, obtained by systematically varying $f_{\text {mask }}$ and $I_{\text {mask }}$. This resembles the type observed experimentally in some cells with a non-monotonic rate-level function (Shamma and Symmes, 1985; Calford and Semple, 1995; Sutter and Loftus, 2003): the field was W-shaped (Fig. $5 g$, red level curves) and surrounded the excitatory RF of the cell (Fig. $5 g$, shaded area). Not surprisingly, the spatial extent along the tonotopic axis of the suppressive RF matched the spatial width of the inhibitory connectivity function $g_{I}(\Delta f)$. The probe response was suppressed by the masker stimulus up to an offset frequency $f_{\text {mask }}$ of $\sim 0.5$ octave with respect to the CF. In contrast, the suppressive region obtained in the CON with the two-tone paradigm was limited almost exclusively to the excitatory RF and its immediate surroundings (data not shown).

Shorter-duration tones produced different RFs. Because there was insufficient time to recruit feedback and recurrent inputs, the responses were shaped primarily by the thalamic input and feedforward inhibition that dominated at the stimulus onset. The response to $f$ near CF showed moderate non-monotonic ratelevel curves (Fig. 5h), whereas tones with large $\Delta f$ produced monotonic rate-level functions. Similar results were obtained with the in vitro LIN network (with no intracortical feedback connections) (Fig. 5i) $(n=6)$. This implies that the minimum LIN model yielding non-monotonic rate-level curves comprises only thalamic inputs and widely spread feedforward inhibition. Auditory subcortical nuclei, such as the inferior colliculus, which contain inhibitory inputs but lack recurrent connections, could rely on a similar feedforward connectivity to generate nonmonotonic rate-level functions.

\section{Response dynamics in the LIN}

The temporal response profiles obtained with the LIN ranged from tonic to phasic, depending on the amount of lateral inhibiin $\boldsymbol{e}$ also applies to $\boldsymbol{f}$. b
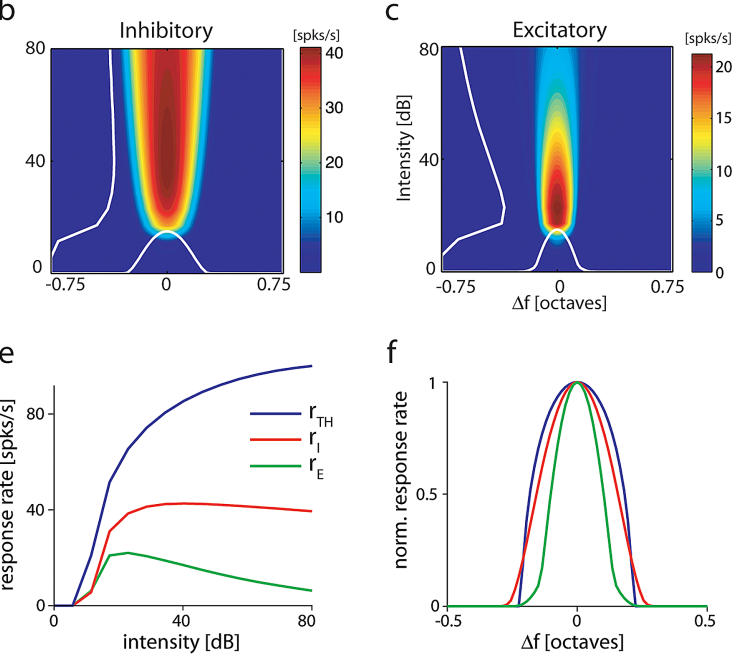

$f$
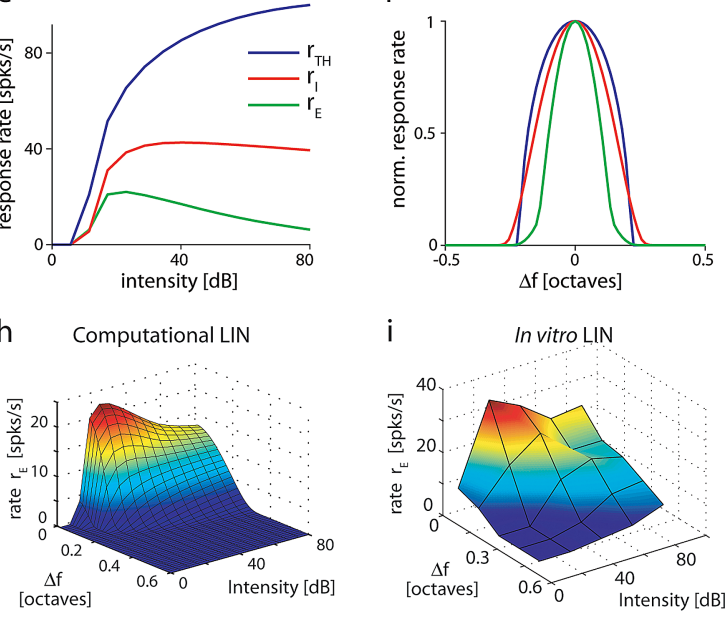

Figure 5. Receptive field properties in the LIN. $\boldsymbol{a}-\boldsymbol{c}$, Receptive fields of a TH $(\boldsymbol{a}), \mathrm{I}(\boldsymbol{b})$, and E (c) cells as in Figure 3. The E cell has non-monotonic rate-level function (white trace on ordinate) and a sharper frequency tuning (white trace on abscissa) than the

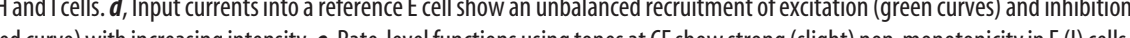

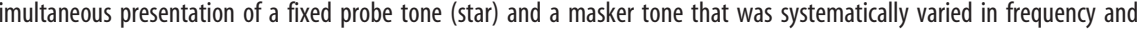
Shaded area shows the RF of the probe alone thresholded at half-height. Because of the recruitment of lateral inhibition by the masker, a prominent suppressive RF flanks the excitatory RF, forming a W shape. $\boldsymbol{h}$, Surface plot of RF obtained with shorter tones monotonic functions. $\boldsymbol{i}$, In vitro network. RF of an $\mathrm{E}$ cell obtained with the in vitro LIN with only feedforward connections. Legend

tion recruited by the stimulus used. The dynamic patterns were quantified by the index $P$ (Eq. 11) that varied continuously from 0 to 1 with increasing intensity and $\Delta f$ (Fig. $6 A a)$. This behavior is fundamentally different from the CON, in which $P$ was approximately invariant over a large range of frequencies and intensities (Fig. 4a).

The response of the E and I cells arose directly from the LIN architecture. When stimulated with a tone at CF and moderate intensity (Fig. $6 A a, \star$ ), the E cell firing was phasic-tonic (Fig. $6 A c$ ). At high intensity (Fig. $6 A a, \mathbf{\square}$ ), the response of $\mathrm{E}$ cells became phasic because more inhibition than excitation was recruited (Fig. 6Ab). Note that the I cell responses remained phasic-tonic at high intensities. A tone at intermediate intensity and $\Delta f>0$ (Fig. $6 A a, 0$ ) elicited a purely phasic response in $\mathrm{E}$ cells (Fig. $6 A e$ ): as $\Delta$ fgrows, the recruitment of inhibition relative to excitation increases, because of the long range connections of I cells along the tonotopic axis. Finally, a tone at CF and low intensity (Fig. $6 A a, \mathbf{\Delta}$ ) elicited an approximately tonic response in both $\mathrm{E}$ and I cells (Fig. 6Ad). This occurred because, at low intensities, little inhibition was recruited and the temporal profile of the response was mainly determined by the tonic thalamic drive. This transition from tonic to phasic-tonic to phasic was greater the larger the degree of non-monotonicity (supplemental 


\section{A. Computational Network}

a
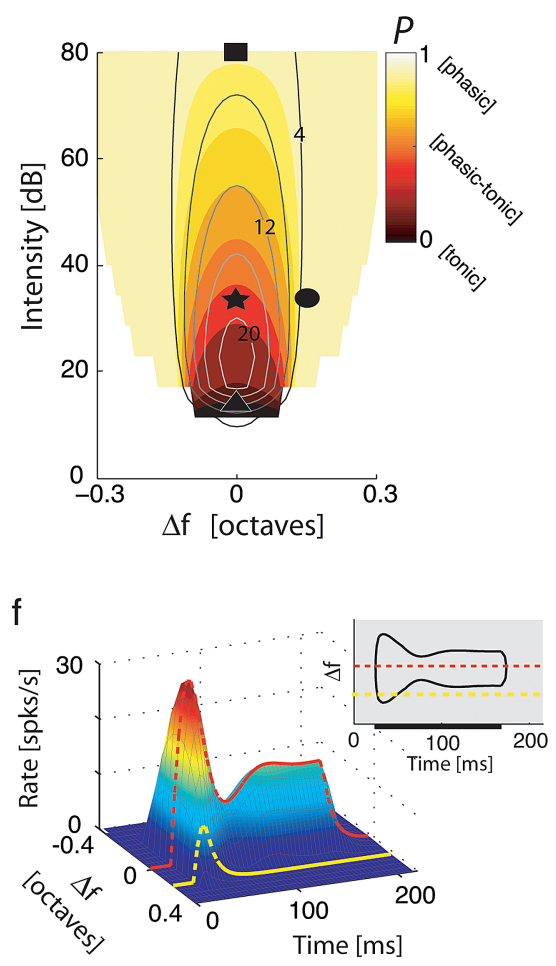

b
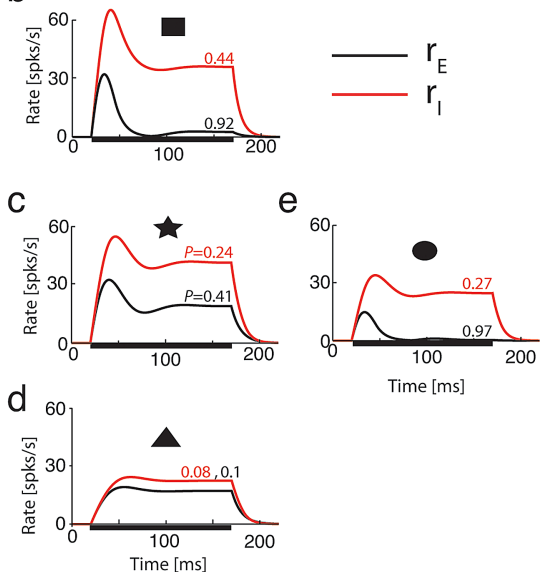

g

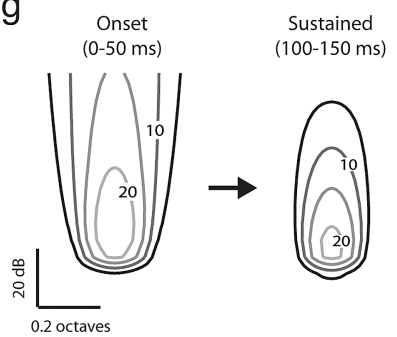

\section{B. In-vitro Network}

a

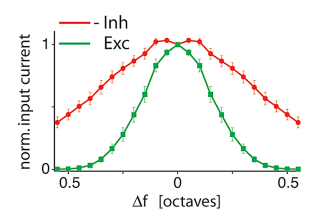

b

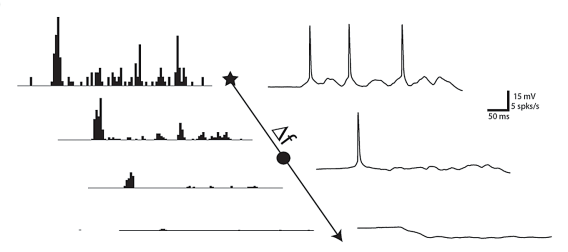

Figure 6. Response dynamics in the LIN. Legend as in Figure 4. A, Computational network. $\boldsymbol{a}$, Map of $P$ index (color) superimposed on RF of an E cell (contour lines). For $f \sim C F$, increasing intensity causes the response to switch from tonic, to phasic-tonic, to phasic. Symbols represent responses highlighted in $\boldsymbol{b}-\boldsymbol{e} . \boldsymbol{b}-\boldsymbol{d}$, Responses of excitatory $\left[r_{\mathrm{E}}(t)\right.$, black $]$ and inhibitory $\left[r_{\mid}(t)\right.$, red $]$ cells to a tone at $C F$ and high $(\boldsymbol{b})$, moderate $(\boldsymbol{c})$, and low $(\boldsymbol{d})$ intensity $(\boldsymbol{\square}, \star$, and $\boldsymbol{\Delta}$ in $\boldsymbol{a}$, respectively). Numbers on the curves indicate $P$ values. $\boldsymbol{e}, r_{\mathrm{E}}(t)$ and $r_{1}(t)$ for a stimulus of moderate intensity and $\Delta f>0(\boldsymbol{i n} \boldsymbol{a})$. $\boldsymbol{f}$, Response pattern of the entire $\mathrm{E}$ population to a $30 \mathrm{~dB}$ tone. Inset shows the iso-rate contour line at half-maximum. $\boldsymbol{g}$, Iso-rate contour lines of the RF of an $\mathrm{E}$ cell computed during the onset (interval, $0-50 \mathrm{~ms}$ ) and sustained (interval, $100-150 \mathrm{~ms}$ ) parts of the response. $\boldsymbol{B}$, In vitro model network. $\boldsymbol{a}$, Normalized average excitatory and inhibitory input currents versus $\Delta f$ into a reference $E$ cell show strong lateral inhibition. $\boldsymbol{b}$, PSTHs (left) and representative voltage traces (right) of an E cell as frequency deviates from (F (top to bottom). Parameters in $\mathbf{A a}-\mathbf{A e}: T=130 \mathrm{~ms} ;(\Delta f, I)=(0,30)(\star),(0,80)(\square),(0,14)(\mathbf{\Delta}),(0.15,30)(\mathbf{O})$, all in octaves and decibels. Parameters in $\boldsymbol{B a}, \boldsymbol{B} \boldsymbol{b}: T=360 \mathrm{~ms} ; \Delta f=0,0.1,0.2$, and 0.3 octaves; PSTH bin size, $5 \mathrm{~ms}$ ( $\boldsymbol{b}$, left); $\Delta f=0,0.2$, and 0.35 octaves (b, right).

Fig. S1f, inset, available at www.jneurosci.org as supplemental material).

The delayed recruitment of inhibition confined the spatial spread of sustained activity in the network to a much narrower frequency band (Fig. $6 \mathrm{Af}$ ): a relatively large fraction of the network was recruited during the transient component (25-50 ms), which then decreased to a smaller pool of cells afterward $(>100$ ms) (Fig. 6Af). Equivalently, stimulation with $f$ at the CF generated phasic-tonic firing of a neuron, which became progressively phasic as $\Delta f$ increased (Fig. $6 A f$, red and yellow dashed tracks, respectively). Some dependence of the response temporal profile on the tone frequency has been observed previously in awake animals (Abeles and Goldstein, 1972; Chimoto et al., 2002; Hro- madka et al., 2008). Specifically, the switch from phasic-tonic to phasic, obtained in the LIN as $\Delta f$ increased, is consistent with the response obtained in awake primates with preferred and nonpreferred stimuli (Wang et al., 2005).

As a result of the LIN response dynamics, the shape of the RF of an E cell evolved dynamically from $\mathrm{V}$-shaped to ovoid (Fig. $6 \mathrm{Ag}$ ). This property has also been documented recently in a fraction of A1 cells in the awake monkey (Sadagopan and Wang, 2008).

The dependence of the firing profile on $\Delta f$ was reproduced in the in vitro LIN. Provided that inhibition had a wider tuning with respect to $f$ (Fig. $6 \mathrm{Ba}$ ), the response was phasic-tonic when $\Delta f \sim 0$ and became purely phasic as $\Delta f$ increased (Fig. $6 B b$ ). Moreover, when $\Delta f$ was sufficiently large to obtain no response, the voltage trace showed a substantial hyperpolarization, a hallmark of lateral inhibition (Fig. $6 \mathrm{Bb}$, bottom voltage trace).

\section{Unbalanced recruitment of excitation and inhibition generates non-monotonicity in the LIN}

To elucidate how excitation and inhibition in the LIN are recruited with increasing intensities, we studied the dependence of the response $r_{\mathrm{E}}$ on the stimulus parameters $A_{\text {in }}$ and $\sigma_{\text {in }}$ (Fig. $\left.7 a\right)$. Recall that the stimulus delivered to the thalamus is Gaussianly distributed along the tonotopic axis with amplitude $A_{\text {in }}$ and SD, $\sigma_{\text {in }}$ (Fig. $2 a$ ). This is important because increasing intensity may be modeled by an increase in $A_{\text {in }}, \sigma_{\text {in }}$, or both (see below). We initially set a threshold-linear thalamic transfer $\phi_{\mathrm{TH}}(s)$ so that variations of $A_{\text {in }}$ or $\sigma_{\text {in }}$ would independently affect the amplitude and the spread of the thalamic activity $r_{\mathrm{TH}}(\Delta f)$, respectively. Increasing $A_{\text {in }}$ at a fixed $\sigma_{\text {in }}$ recruited $\mathrm{E}$ and I populations proportionally so that $r_{\mathrm{E}}$ increased monotonically (Fig. $7 a$, straight arrow). In contrast, increasing $\sigma_{\text {in }}$ of the input at a fixed $A_{\text {in }}$ produced non-monotonic changes in $r_{\mathrm{E}}$ (Fig. 7a, curved arrow). Similar behavior was reproduced with the in vitro LIN network ( $n=7$ experiments), demonstrating its generality and robustness (Fig. $7 b$ ).

The non-monotonic response obtained with increasing $\sigma_{\text {in }}$ is a consequence of the different widths of the connectivity functions $g_{\mathrm{TH}}(\Delta f)$ and $g_{\mathrm{I}}(\Delta f)$ (Fig. 7c). For simplicity, we first limit the analyses to models with only feedforward connections. As input widened (increasing $\sigma_{\text {in }}$ ) (Fig. $7 c$, from left to right), both $\mathrm{TH}$ neurons and I cortical cells were recruited. The profile of the population responses $r_{\mathrm{TH}}$ and $r_{\mathrm{I}}$ are indicated by the gray shaded areas (Fig. $7 c$ ). When the input width surpasses that of $g_{\mathrm{TH}}(\Delta f)$ (Fig. $7 c$, middle bottom), all available presynaptic thalamic cells converging to a reference cortical $\mathrm{E}$ cell will have been recruited, 
a

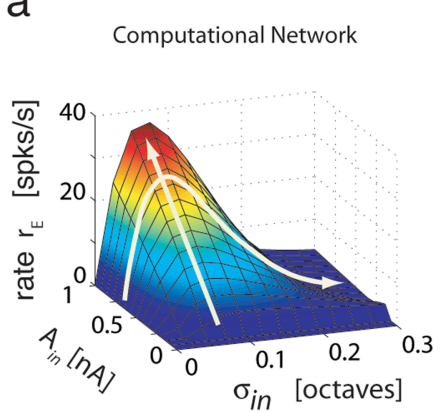

C

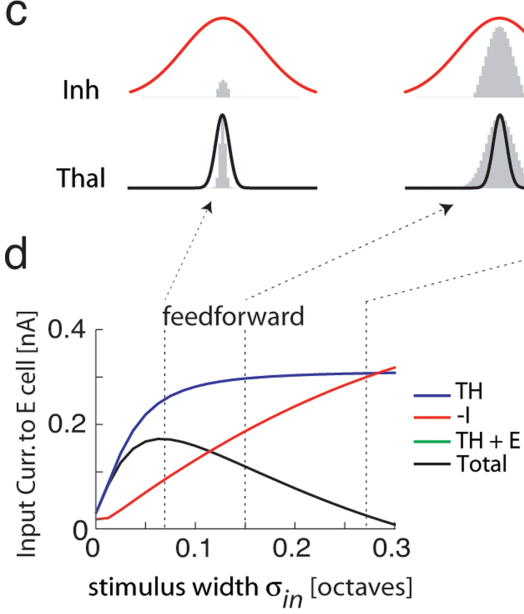

f
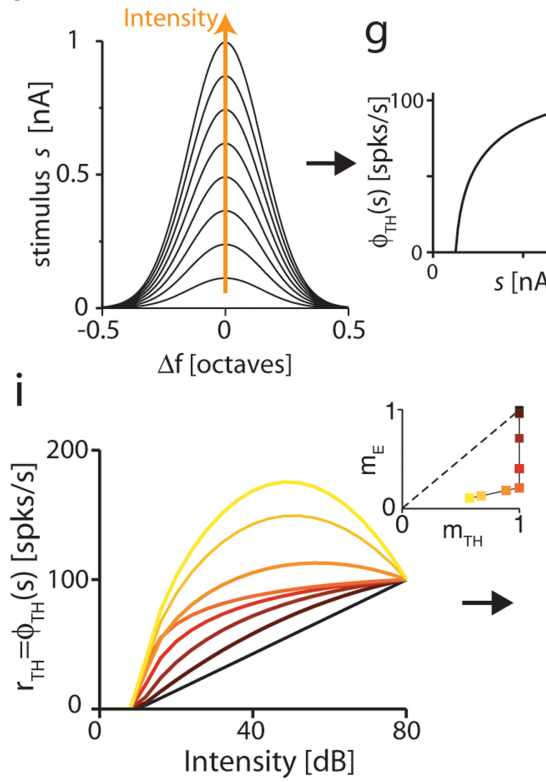

j

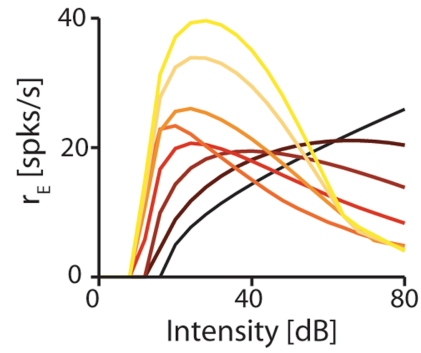

Figure 7. Unbalanced recruitment of excitation and inhibition in the LIN. $\boldsymbol{a}$, Computational network. Surface plot of the response rate $r_{\mathrm{E}}$ as a function of the amplitude $A_{\text {in }}$ and spread of the thalamic input $\sigma_{\text {in }}$ when using a threshold-linear thalamic transfer function $\phi_{\mathrm{TH}}(s)$ (see Results). The response $r_{\mathrm{E}}$ increases monotonically with $A_{\text {in }}$ and non-monotonically with $\sigma_{\text {in }}$ (white arrows). $\boldsymbol{b}$, In vitro network. This result was reproduced using the in vitro LIN in which increasing intensity was modeled as either an ad hoc simultaneous increase in $A_{\text {in }}$ and $\sigma_{\text {in }}$ (orange track) or an increase in $A_{\text {in }}$ followed by an increase in $\sigma_{\text {in }}$ (white track). Both alternatives yield a non-monotonic rate-intensity function (inset). $c$, Thalamic population activity $\left(r_{\mathrm{TH}}(\Delta f)\right.$, bottom shaded areas) and inhibitory population activity $\left[r_{1}(\Delta f)\right.$, top shaded areas] superimposed on the connectivity functions $g_{\mathrm{TH}}(\Delta f)$ (black) and $g_{\mid}(\Delta f)$ (red) for three values of the stimulus spread. For details, see Results. $\boldsymbol{d}$, Plot of individual currents generated in a reference $E$ cell versus stimulus spread (examples in c correspond to those points indicated by dashed lines in $\boldsymbol{d}$ ). $\boldsymbol{e}$, Similar non-monotonic functions were obtained in an LIN network with intracortical feedback, although the individual currents behaved differently (see Results).f, Tone intensity was encoded in the amplitude $A_{\text {in }}$ (at a fixed $\sigma_{\text {in }}$ ) of the stimulus function (Eq. 4).g, Using a nonlinear concave thalamic response function $\phi_{\mathrm{TH}}(s)$ causes the amplitude and spread of the thalamic activity to increase with intensity $(\boldsymbol{h}), \boldsymbol{i}, \boldsymbol{j}$, Decreasing the degree of concavity of $\phi_{\mathrm{TH}}(s)$ reduces the degree of non-monotonicity of the rate-level function. producing a morphological saturation of the thalamic input. Conversely, the larger width of $g_{\mathrm{I}}(\Delta f)$ means that more presynaptic inhibitory cells can still be recruited with additional increases in $\sigma_{\text {in }}$ (Fig. $7 c$, top right). Consequently, the inhibitory current increased almost linearly with $\sigma_{\text {in }}$, whereas the thalamic current quickly saturates, yielding a non-monotonically increasing total current in the reference $\mathrm{E}$ cell (Fig. $7 d$ ).

Adding recurrent excitatory feedback IE and EE connections produced similar results. The main effect was to initially increase the excitatory drive to both $\mathrm{E}$ and I populations (Fig. $7 e$, green). At small $\sigma_{\text {in }}$, the $\mathrm{TH}$ input into the reference $\mathrm{E}$ cell had not saturated and the recurrent connections added substantial amounts of $\mathrm{E}$ and I input currents into the reference E cell. For larger $\sigma_{\text {in }}$, the TH input saturated and inhibition dominated, thereby suppressing the response of the E population. This yielded a non-monotonic excitatory input into the reference E cell (green). This behavior was robust over a wide range of parameters in the LIN and is similar to what has been observed recently in in vivo whole-cell recordings, in which the excitatory but not the inhibitory conductance increased non-monotonically with intensity (Wu et al., 2006). Our analyses suggest that the non-monotonicity can arise from the nonlinear dynamics of a cortical network even when TC inputs are monotonic.

A sufficient condition for obtaining non-monotonic responses is therefore that $\sigma_{\text {in }}$ increases with the stimulus intensity. Many trajectories involving an increase in both amplitude and spread would yield a non-monotonic response rate such as a simultaneous linear increase in both $A_{\text {in }}$ and $\sigma_{\text {in }}$, or an increase in $A_{\text {in }}$ followed by an increase in $\sigma_{\text {in }}$ (Fig. $7 b$, white and orange tracks, respectively).

However, non-monotonic rate level functions can also be produced without a change in $\sigma_{\text {in }}$, if the nonlinearities of the thalamic response function $\phi_{\mathrm{TH}}(s)$ are taken into account (Fig. $7 g, i$ ). Here, the function $\phi_{\mathrm{TH}}(s)$ must be interpreted as the rate-level function of thalamic cells, which show different degrees of saturation and

$\leftarrow$

A monotonic function is obtained when $\phi_{\mathrm{TH}}(s)$ becomes linear (black). Making $\phi_{\mathrm{TH}}(s)$ slightly non-monotonic sharpened the intensity tuning in cortex (yellow curves). This sharpening is summarized in the $m_{\mathrm{TH}} \rightarrow m_{\mathrm{E}}$ mapping shown in the inset. Parameters in $\boldsymbol{c}-\boldsymbol{e}: A_{\text {in }}=0.5 \mathrm{nA} ; \boldsymbol{f}-\boldsymbol{h}$ : intensity varies from 0 to $80 \mathrm{~dB}$ in $10 \mathrm{~dB}$ steps; $\boldsymbol{i}, \boldsymbol{j}$ : concavity varies from yellow to brown $(b, c)=(4,0.2),(2.7,0.2),(1,0.2),(0,0.5),(0,0.1)$, $(0,0.02),(0,0.001)$ and linear; $c=0.2$ in the rest; $f=C F$. 
non-monotonicity (Katsuki et al., 1958; Aitkin and Webster, 1972; Rouiller et al., 1983). This is modeled by modifying $\phi_{\mathrm{TH}}(s)$ to have either a monotonic concave shape or slight degrees of non-monotonicity (Fig. $7 g, i)$. The net result of these nonlinearities is that the thalamic response $r_{\mathrm{TH}}(\Delta f)$ increased nonmultiplicatively with intensity so that both the amplitude and the spread of inputs to the cortical layer increased (Fig. $7 f-h$ ). This has the same qualitative effect of increasing both $A_{\text {in }}$ and $\sigma_{\text {in }}$, that is, to recruit excitation and inhibition in an unbalanced manner. Moreover, this was the mechanism by which an unbalanced recruitment of excitation and inhibition was obtained in Figures 5 and 6 . In the in vitro simulations, $A_{\text {in }}$ and $\sigma_{\text {in }}$ were varied ad hoc with increasing intensity after either of the trajectories shown in Figure $7 b$ (white and orange tracks).

Increasing the degree of concavity of $\phi_{\mathrm{TH}}(s)$ from linear to strongly saturating caused the cortical rate-level functions to rapidly change from monotonic to non-monotonic (Fig. 7i,j). Furthermore, making $\phi_{\mathrm{TH}}(s)$ slightly non-monotonic resulted in a strong non-monotonicity in the cortical response (Fig. 7i,j, yellow curves). In summary, the unbalanced recruitment of excitation and inhibition in an LIN was a robust mechanism to generate non-monotonic rate-level functions provided that the input current into AI widened with increasing intensity. Alternatively, lateral inhibition could sharpen an already existing non-monotonic TC input, thus enhancing the intensity tuning of AI cells with respect to MGv cells (Fig. 7i, inset). This sharpening of the intensity tuning stands in contrast with the behavior of the CON in which $m_{\mathrm{E}}=m_{\mathrm{TH}}$ (dashed line in Fig. $7 d$, inset) such that (1) monotonic inputs yield monotonic outputs, and (2) nonmonotonic inputs yield outputs with the exact same degree of non-monotonicity (data not shown).

The different response properties obtained in the LIN are compared with those obtained in the CON in Table 1. In the supplemental data (available at www.jneurosci.org as supplemental material), we extend the analysis of the LIN using tones with different rise times and broadband noise inputs (supplemental Fig. S5, available at www.jneurosci.org as supplemental material).

\section{Discussion}

We used a combination of computational analysis and in vitro experiments to show that thalamocortical network architecture can account for many of the properties of AI neural responses documented in vivo. We were motivated by the finding that, in some layer 4 AI cells, the PSPs evoked by thalamic stimulation in RS cells varied non-monotonically with the intensity of the stimulus delivered to MGv (Fig. 1). We developed a model for the MGv-AI circuitry that was implemented computationally using simple rate cell units (Wilson and Cowan, 1972) and in vitro by simulating the network activity using neurons recorded from cortical slices (Reyes, 2003). Both the computational and the in vitro networks produced similar results, indicating that the phenomena are a general consequence of the network properties and do not depend substantially on specific neuron properties or model details. Indeed, the results were valid for a relatively wide parameter space, and no fine-tuning was needed.

\section{CON versus LIN}

Our results indicate that CON and LIN have properties that are distinct from each other (see summary in Table 1). By construction, the excitatory and inhibitory inputs to a reference cell in the CON vary proportionally with changing frequencies and intensities. The tight relationship between E and I produced (1) cortical RFs that inherit the shape of thalamic RF (Fig. $3 a-c$ ), (2) no sharpening of the frequency tuning (Fig. 3f), and (3) monotonic rate-level functions
(Fig. 3e) (but see supplemental Fig. S4d-f, available at www. jneurosci.org as supplemental material). In contrast, the excitatory and inhibitory inputs to a reference cell in the LIN are primarily unbalanced; inhibition dominates at stimulus frequencies far from $\mathrm{CF}$ and at high intensities. This differential recruitment of excitatory and inhibitory inputs results in (1) cortical RFs that differ substantially from thalamic RFs (Fig. 5a-c), (2) clear sideband inhibition that sharpens $f$ tuning curves (Fig. $5 f$ ), and (3) strong (weak) nonmonotonic rate-level functions for $\mathrm{E}$ (I) cells (Fig. 5e). The LIN further reproduces experimental results showing that the degree of non-monotonicity of E cells increases with decreasing tone rise times (supplemental Fig. S5c,d) (Phillips, 1988; Phillips et al., 1995; Heil and Irvine, 1998) and that E cells with non-monotonic functions are almost unresponsive to wideband noise stimuli (supplemental Fig. $\mathrm{S} 5 c, d)$. It also predicts an increasing dependence of the nonmonotonicity with the tone duration (supplemental Fig. S5a, available at www.jneurosci.org as supplemental material).

There is some debate as to whether $\mathrm{AI}$ is configured as $\mathrm{CON}$ or LIN, and experimental evidence supporting one or the other is still inconclusive. Recent whole-cell recordings in vivo seem to support the CON as excitatory, and inhibitory conductance recorded in a reference cell covaried with frequency and intensity (Wehr and Zador, 2003; Tan et al., 2004). However, a recent study has showed that the cotuning is only approximate and that the inhibition is broader than the excitation (Wu et al., 2008). The results in Figures 5-7 are robust and can be reproduced using a modified LIN whose inhibitory inputs are only slightly broader than excitatory inputs (see supplemental data, available at www.jneurosci.org as supplemental material). Intracellular recordings also show that the inputs to cells can become unbalanced with increasing intensity (Ojima and Murakami, 2002; Wu et al., 2006; Tan et al., 2007). Indeed, the experiments shown in Figure 1 show that, in a fraction of cells, inhibition is recruited in an unbalanced manner at relatively high stimulus intensities.

Unbalanced excitatory and inhibitory inputs yielding nonmonotonic rate-level curves can be obtained in a CON circuit in two ways. First, if the "effective" firing threshold of I cells is much higher than that of E cells, then there exists a range of low tone intensities in which E cells respond but I cells remain subthreshold (supplemental Fig. S $4 c-e$, available at www.jneurosci.org as supplemental material). This seems unlikely because TC PSPs simultaneously obtained in vitro through stimulation of MGv are on average two times larger on FS cells than in RS cells over a large range different intensities but specifically at low intensities (M. Schiff and A. Reyes, unpublished observation) (compare, for instance, the dark brown traces shown in Fig. 1b). This difference in TC PSPs amplitudes, which has also been observed in barrel cortex (Sun et al., 2006; Cruikshank et al., 2007), is consistent with in vivo studies showing that FS cells have a lower intensity threshold than RS cells (Atencio and Schreiner, 2008; Wu et al., 2008).

Second, a CON network could yield non-monotonic responses if these were simply inherited from non-monotonic inputs from the thalamus. However, to be consistent with recent in vivo data (Wu et al., 2006; Tan et al., 2007), E and I cells would have to inherit their responses from different subpopulations of MGv afferents: inputs onto $\mathrm{E}$ would be have to be nonmonotonic, whereas those onto I cells would have to be monotonic. Again, this seems unlikely because in vivo recordings show that IPSPs follow EPSPs at a fixed delay with very little variability (Wehr and Zador, 2003): more variability would be expected if excitatory and inhibitory cells receive independent sources of TC inputs. Moreover, in vitro recordings in both somatosensory cortex (Inoue and Imoto, 2006) and AI (Schiff and Reyes, unpub- 
lished observation) indicate that a large portion of inputs into excitatory and inhibitory cells are likely to arise from a common set of TC afferents.

Unbalanced recruitment of excitation and inhibition yielding non-monotonic rate-level curves is more robustly obtained in an LIN (Figs. $5 e, 7 j$ ). An LIN circuit also produced a pattern of input currents like the one found in in vivo whole-cell recordings (Wu et al., 2006; Tan et al., 2007) without segregating the TC afferents into E and I populations (Figs. $5 d, 7 e$ ). In addition, the responses obtained in the LIN were consistent with a number of experimental observations: first, extracellularly recorded responses to acoustic stimuli show that some neurons in AI exhibit suppressive RFs that exceed the width of the excitatory RFs (Shamma and Symmes, 1985; Calford and Semple, 1995; Brosch and Schreiner, 1997; Sutter et al., 1999; Sutter and Loftus, 2003). Second, the temporal response profile of AI cells can be tonic or phasic, depending on whether the stimulus is preferred or nonpreferred, respectively (Wang et al., 2005). Third, RFs of E cells were much narrower than the RF of the synaptic thalamic inputs (Liu et al., 2007) but in the CON had the same width (data not shown). This suggests that this sharpening is attributable to lateral inhibition and not to boosting via recurrent excitatory connections (Liu et al., 2007), which were present in both the LIN and CON. Finally, an LIN connectivity seems the more plausible explanation for those cells in our data showing a nonmonotonic behavior of subthreshold TC synaptic potentials caused by an unbalanced recruitment of RS and FS cells (Fig. 1).

In a recent study conducted in awake primates, Sadagopan and Wang (2008) classified A1 cells having a "V"/"I"-shaped RF or a closed "O"-shaped RF. The latter category shows a strongly nonmonotonic rate-level function and a much narrower bandwidth reminiscent of cells in the LIN. Interestingly, they found that $\sim 65 \%$ of the cells had closed RFs, similar to other studies in awake monkey ( $\sim 80 \%$ non-monotonic cells in the study by Pfingst and O'Connor, 1981) but a much greater fraction than previous studies performed in anesthetized animals [ 5\% in rat A1 (Wu et al., 2006); 25\% in cat A1 (Phillips et al., 1989; Heil et al., 1994; Sutter and Schreiner, 1995)], indicating that anesthesia has a large impact on the RF shape of cells in A1. This prompts a reassessment of the intracellular data quoted above which was obtained in anesthetized rats and suggests that non-monotonic rate-level functions are far more abundant in the behaving auditory cortex than previously thought.

Despite the enormous amount of work on the characterization of neural responses in AI, there have been few computational studies about the cortical circuits underlying these responses (Nomoto, 1979; Dominguez et al., 2006; Soto et al., 2006). Our model gives the minimal conditions that give rise to some basic response properties observed in AI. The addition of other features such as short-term synaptic depression and facilitation in both TC (Castro-Alamncos, 1997; Rose and Metherate, 2005) and intracortical connections (Atzori et al., 2001; Oswald and Reyes, 2008) would provide insight into the responses to more complex stimuli such as trains of clicks (De Ribaupierre, 1972; Creutzfeldt et al., 1980; Eggermont, 1991; Lu et al., 2001) or to forward masking experiments, in which the timescales of response suppression can last hundreds of milliseconds (Eggermont, 1999; Denham, 2001; Wehr and Zador, 2005).

No single connectivity pattern is likely to explain the variety of different intensity-frequency RF shapes observed in AI, and it seems more plausible that several patterns of connectivity cooccur. We have separately studied how two of these patterns, LIN and CON, influence the response properties of AI cells. These architectures should be viewed, however, as limit cases of a potential continuum of connectivity patterns going from $\mathrm{CON}$ to
LIN. Future work should address how these two connectivity patterns may coexist in the same network without disrupting the properties of each subpopulation and which synaptic changes could turn the response of some monotonic cells into nonmonotonic (Polley et al., 2004).

\section{References}

Abeles M, Goldstein MH Jr (1972) Responses of single units in the primary auditory cortex of the cat to tones and to tone pairs. Brain Res 42:337-352.

Aitkin LM, Webster WR (1972) Medial geniculate body of the cat: organization and responses to tonal stimuli of neurons in ventral division. J Neurophysiol 35:365-380.

Atencio CA, Schreiner CE (2008) Spectrotemporal processing differences between auditory cortical fast-spiking and regular-spiking neurons. J Neurosci 28:3897-3910.

Atzori M, Lei S, Evans DI, Kanold PO, Phillips-Tansey E, McIntyre O, McBain CJ (2001) Differential synaptic processing separates stationary from transient inputs to the auditory cortex. Nat Neurosci 4:1230-1237.

Ben-Yishai R, Bar-Or RL, Sompolinsky H (1995) Theory of orientation tuning in visual cortex. Proc Natl Acad Sci U S A 92:3844-3848.

Brosch M, Schreiner CE (1997) Time course of forward masking tuning curves in cat primary auditory cortex. J Neurophysiol 77:923-943.

Brugge JF, Dubrovsky NA, Aitkin LM, Anderson DJ (1969) Sensitivity of single neurons in auditory cortex of cat to binaural tonal stimulation; effects of varying interaural time and intensity. J Neurophysiol 32:1005-1024.

Calford MB, Semple MN (1995) Monaural inhibition in cat auditory cortex. J Neurophysiol 73:1876-1891.

Castro-Alamancos MA (1997) Short-term plasticity in thalamocortical pathways: cellular mechanisms and functional roles. Rev Neurosci 8:95-116.

Chen QC, Jen PH (2000) Bicuculline application affects discharge patterns, rate-intensity functions, and frequency tuning characteristics of bat auditory cortical neurons. Hear Res 150:161-174.

Chimoto S, Kitama T, Qin L, Sakayori S, Sato Y (2002) Tonal response patterns of primary auditory cortex neurons in alert cats. Brain Res 934:34-42.

Creutzfeldt O, Hellweg FC, Schreiner C (1980) Thalamocortical transformation of responses to complex auditory stimuli. Exp Brain Res 39:87-104.

Cruikshank SJ, Rose HJ, Metherate R (2002) Auditory thalamocortical synaptic transmission in vitro. J Neurophysiol 87:361-384.

Cruikshank SJ, Lewis TJ, Connors BW (2007) Synaptic basis for intense thalamocortical activation of feedforward inhibitory cells in neocortex. Nat Neurosci 10:462-468.

De Ribaupierre F, Goldstein MH Jr, Yeni-Komshian G (1972) Intracellular study of the cat's primary auditory cortex. Brain Res 48:185-204.

Denham SL (2001) Cortical synaptic depression and auditory perception. In: Computational models of auditory function (Greenberg S and Slaney M, eds), pp 281-296. Amsterdam: IOS.

DeWeese MR, Wehr M, Zador AM (2003) Binary spiking in auditory cortex. J Neurosci 23:7940-7949.

Dominguez M, Becker S, Bruce I, Read H (2006) A spiking neuron model of cortical correlates of sensorineural hearing loss: spontaneous firing, synchrony, and tinnitus. Neural Comp 18:2942-2958.

Eggermont JJ (1991) Rate and synchronization measures of periodicity coding in cat primary auditory cortex. Hear Res 56:153-167.

Eggermont JJ (1999) The magnitude and phase of temporal modulation transfer functions in cat auditory cortex. J Neurosci 19:2780-2788.

Ehret G, Merzenich MM (1988) Complex sound analysis (frequency resolution, filtering and spectral integration) by single units of the inferior colliculus of the cat. Brain Res Rev 13:139-163.

Evans EF, Whitfield IC (1964) Classification of unit responses in the auditory cortex of the unanaesthetized and unrestrained cat. J Physiol 171:476-493.

Greenwood DD, Maruyama N (1965) Excitatory and inhibitory response areas of auditory neurons in the cochlear nucleus. J Neurophysiol 28:863-892.

Heil P, Irvine DR (1998) The posterior field P of cat auditory cortex: coding of envelope transients. Cereb Cortex 8:125-141.

Heil P, Rajan R, Irvine DRF (1994) Topographic representation of tone in- 
tensity along the isofrequency axis of cat primary auditory cortex. Hearing Res 76:188-202.

Hromádka T, DeWeese MR, Zador AM (2008) Sparse representation of sounds in the unanesthetized auditory cortex. PLoS Biol 6:e16.

Inoue T, Imoto K (2006) Feedforward inhibitory connections from multiple thalamic cells to multiple regular-spiking cells in layer 4 of the somatosensory cortex. J Neurophysiol 96:1746-1754.

Jen PH, Chen QC, Wu FJ (2002) Interaction between excitation and inhibition affects frequency tuning curve, response size and latency of neurons in the auditory cortex of the big brown bat, Eptesicus fuscus. Hear Res 174:281-289.

Katsuki Y, Sumi T, Uchiyama H, Watanabe T (1958) Electric responses of auditory neurons in cat to sound stimulation. J Neurophysiol 21:569-588.

Kilgard MP, Merzenich MM (1999) Distributed representation of spectral and temporal information in rat primary auditory cortex. Hearing Res 134:16-28.

Liu BH, Wu GK, Arbuckle R, Tao HW, Zhang LI (2007) Defining cortical frequency tuning with recurrent excitatory circuitry. Nat Neurosci 10:1594-1600.

Lu T, Liang L, Wang X (2001) Temporal and rate representations of timevarying signals in the auditory cortex of awake primates. Nat Neurosci 4:1131-1138.

Mendelson JR, Cynader MS (1985) Sensitivity of cat primary auditory cortex (AI) neurons to the direction and rate of frequency modulation. Brain Res 327:331-335.

Nomoto M (1979) Spatial firing patterns of auditory neuron network modelling by computer simulation. Biol Cybern 32:227-237.

Ojima H, Murakami K (2002) Intracellular characterization of suppressive responses in supragranular pyramidal neurons of cat primary auditory cortex in vivo. Cereb Cortex 12:1079-1091.

Oswald AM, Reyes AD (2008) Maturation of intrinsic and synaptic properties of layer $2 / 3$ pyramidal neurons in mouse auditory cortex. J Neurophysiol 99:2998-3008.

Oswald AM, Schiff ML, Reyes AD (2006) Synaptic mechanisms underlying auditory processing. Curr Opin Neurobiol 16:371-376.

Pfingst BE, O'Connor TA (1981) Characteristics of neurons in auditory cortex of monkeys performing a simple auditory task. J Neurophysiol 45:16-34.

Phillips DP (1988) Effect of tone-pulse rise time on rate-level functions of cat auditory cortex neurons: excitatory and inhibitory processes shaping responses to tone onset. J Neurophysiol 59:1524-1539.

Phillips DP (1990) Neural representation of sound amplitude in the auditory cortex: effects of noise masking. Behav Brain Res 37:197-214.

Phillips DP, Orman SS, Musicant AD, Wilson GF (1985) Neurons in the cat's primary auditory cortex distinguished by their responses to tones and wide-spectrum noise. Hear Res 18:73-86.

Phillips DP, Hall SE, Hollett JL (1989) Repetition rate and signal level effects on neuronal responses to brief tone pulses in cat auditory cortex. J Acoust Soc Am 85:2537-2549.

Phillips DP, Semple MN, Kitzes LM (1995) Factors shaping the tone level sensitivity of single neurons in posterior field of cat auditory cortex. J Neurophysiol 73:674-686.

Phillips DP, Hall SE, Boehnke SE (2002) Central auditory onset responses, and temporal asymmetries in auditory perception. Hear Res 167:192-205.

Pinto DJ, Hartings JA, Brumberg JC, Simons DJ (2003) Cortical damping: analysis of thalamocortical response transformations in rodent barrel cortex. Cereb Cortex 13:33-44.

Polley DB, Heiser MA, Blake DT, Schreiner CE, Merzenich MM (2004) Associative learning shapes the neural code for stimulus magnitude in primary auditory cortex. Proc Natl Acad Sci U S A 101:16351-16356.

Recanzone GH (2000) Response profiles of auditory cortical neurons to tones and noise in behaving macaque monkeys. Hear Res 150:104-118.

Reyes A, Lujan R, Rozov A, Burnashev N, Somogyi P, Sakmann B (1998) Target-cell-specific facilitation and depression in neocortical circuits. Nat Neurosci 1:279-285.

Reyes AD (2003) Synchrony-dependent propagation of firing rate in iteratively constructed networks in vitro. Nat Neurosci 6:593-599.

Rose HJ, Metherate R (2005) Auditory thalamocortical transmission is reliable and temporally precise. J Neurophysiol 94:2019-2030.

Rouiller E, de Ribaupierre Y, Morel A, de Ribaupierre F (1983) Intensity functions of single unit responses to tone in the medial geniculate body of cat. Hear Res 11:235-247.

Sadagopan S, Wang X (2008) Level invariant representation of sounds by populations of neurons in primary auditory cortex. J Neurosci 28:3415-3426.

Sally SL, Kelly JB (1988) Organization of auditory cortex in the albino rat: sound frequency. J Neurophysiol 59:1627-1638.

Schreiner CE, Mendelson JR, Sutter ML (1992) Functional topography of cat primary auditory cortex: representation of tone intensity. Exp Brain Res 92:105-122.

Schreiner CE, Read HL, Sutter ML (2000) Modular organization of frequency integration in primary auditory cortex. Annu Rev Neurosci 23:501-529.

Shamma SA, Symmes D (1985) Patterns of inhibition in auditory cortical cells in awake squirrel monkeys. Hear Res 19:1-13.

Soto G, Kopell N, Sen K (2006) Network architecture, receptive fields, and neuromodulation: computational and functional implications of cholinergic modulation in primary auditory cortex. J Neurophysiol 96:2972-2983.

Suga N (1971) Responses of inferior collicular neurones of bats to tone bursts with different rise times. J Physiol 217:159-177.

Suga N (1977) Amplitude spectrum representation in the Dopplershifted-CF processing area of the auditory cortex of the mustache bat. Science 196:64-67.

Suga N (1995) Sharpening of frequency tuning by inhibition in the central auditory system: tribute to Yasuji Katsuki. Neurosci Res 21:287-299.

Suga N, Manabe T (1982) Neural basis of amplitude-spectrum representation in auditory cortex of the mustached bat. J Neurophysiol 47:225-255.

Sun QQ, Huguenard JR, Prince DA (2006) Barrel cortex microcircuits: thalamocortical feedforward inhibition in spiny stellate cells is mediated by a small number of fast-spiking interneurons. J Neurosci 26:1219-1230.

Sutter ML, Loftus WC (2003) Excitatory and inhibitory intensity tuning in auditory cortex: evidence for multiple inhibitory mechanisms. J Neurophysiol 90:2629-2647.

Sutter ML, Schreiner CE (1995) Topography of intensity tuning in cat primary auditory cortex: single-neuron versus multiple-neuron recordings. J Neurophysiol 73:190-204.

Sutter ML, Schreiner CE, McLean M, O’Connor KN, Loftus WC (1999) Organization of inhibitory frequency receptive fields in cat primary auditory cortex. J Neurophysiol 82:2358-2371.

Tan AY, Zhang LI, Merzenich MM, Schreiner CE (2004) Tone-evoked excitatory and inhibitory synaptic conductances of primary auditory cortex neurons. J Neurophysiol 92:630-643.

Tan AY, Atencio CA, Polley DB, Merzenich MM, Schreiner CE (2007) Unbalanced synaptic inhibition can create intensity-tuned auditory cortex neurons. Neuroscience 146:449-462.

Ulanovsky N, Las L, Nelken I (2003) Processing of low-probability sounds by cortical neurons. Nat Neurosci 6:391-398.

Volkov IO, Galazjuk AV (1991) Formation of spike response to sound tones in cat auditory cortex neurons: interaction of excitatory and inhibitory effects. Neuroscience 43:307-321.

Wang J, McFadden SL, Caspary D, Salvi R (2002) Gamma-aminobutyric acid circuits shape response properties of auditory cortex neurons. Brain Res 944:219-231.

Wang X, Lu T, Snider RK, Liang L (2005) Sustained firing in auditory cortex evoked by preferred stimuli. Nature 435:341-346.

Wehr M, Zador AM (2003) Balanced inhibition underlies tuning and sharpens spike timing in auditory cortex. Nature 426:442-446.

Wehr M, Zador AM (2005) Synaptic mechanisms of forward suppression in rat auditory cortex. Neuron 47:437-445.

Wilson HR, Cowan JD (1972) Excitatory and inhibitory interactions in localized populations of model neurons. Biophys J 12:1-24.

Wu GK, Li P, Tao HW, Zhang LI (2006) Nonmonotonic synaptic excitation and imbalanced inhibition underlying cortical intensity tuning. Neuron 52:705-715.

Wu GK, Arbuckle R, Liu BH, Tao HW, Zhang LI (2008) Lateral sharpening of cortical frequency tuning by approximately balanced inhibition. Neuron 58:132-143.

Young ED, Brownell WE (1976) Responses to tones and noise of single cells in dorsal cochlear nucleus of unanesthetized cats. J Neurophysiol 39:282-300.

Zhang LI, Tan AY, Schreiner CE, Merzenich MM (2003) Topography and synaptic shaping of direction selectivity in primary auditory cortex. Nature 424:201-205. Vol. 28, No. 35 